\title{
A Relativistic DFT Probe for Reaction Energies and Electronic/Bonding Properties of Polypyrrolic Heterobimetallic Actinide Complexes: Effects of Uranyl endo-oxo
}

\section{Functionalization}

\author{
Xiu-Jun Zheng ${ }^{a^{*}}$, Raza Ullah Shah Bacha ${ }^{b}$, Dong-Mei Su ${ }^{c}$ and Qing-Jiang Pan ${ }^{b^{*}}$ \\ ${ }^{a}$ Institute of Food and Environmental Engineering, East University of Heilongiiang, Harbin,
} 150066, China.

${ }^{\mathrm{b}}$ Key Laboratory of Functional Inorganic Material Chemistry, School of Chemistry and Materials Science, Heilongjiang University, Harbin, 150080, China.

${ }^{c}$ State-owned Assets Management Division, Harbin University, Harbin, 150086, China

Corresponding Authors

zhengxiujun2004@163.com (X.-J. Z.)

panqjitc@163.com (Q.-J .P.) 


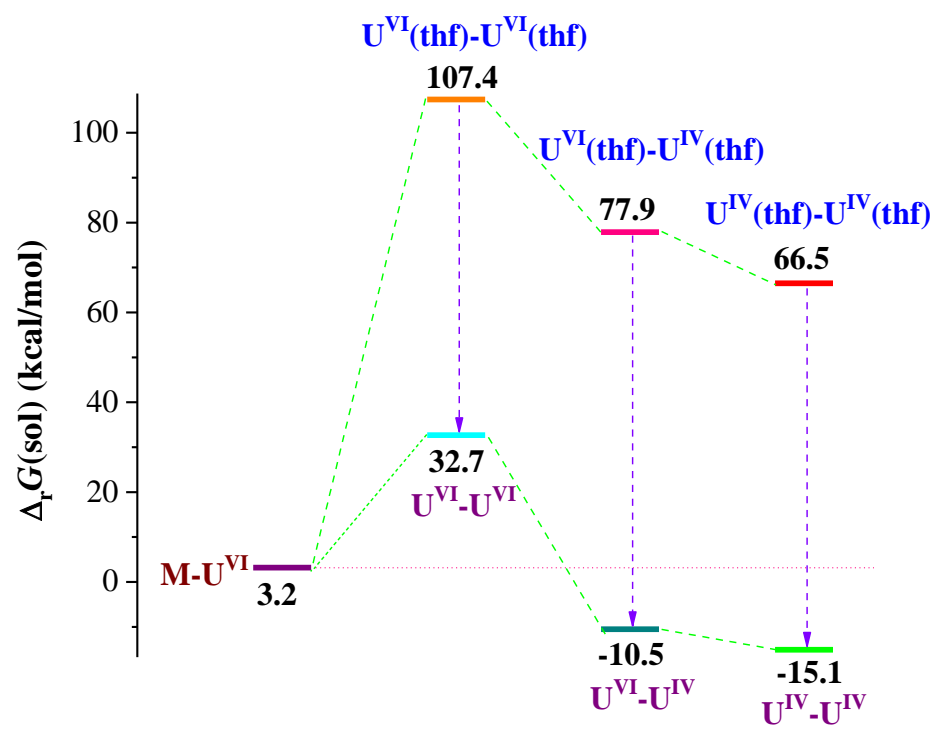

Figure S1. Free energies $\Delta_{\mathrm{r}} G\left(\right.$ sol) of formation reactions of $\mathbf{U}^{\mathrm{VI}}(\mathbf{t h f})-\mathbf{U}^{\mathrm{VI}}(\mathbf{t h f}), \mathbf{U}^{\mathrm{VI}}(\mathbf{t h f})-\mathbf{U}^{\mathrm{IV}}($ thf $)$, $\mathbf{U}^{\mathrm{IV}}($ thf $)-\mathbf{U}^{\mathrm{IV}}($ thf $), \mathbf{U}^{\mathrm{VI}}-\mathbf{U}^{\mathrm{VI}}, \mathbf{U}^{\mathrm{VI}}-\mathbf{U}^{\mathrm{IV}}, \mathbf{U}^{\mathrm{IV}}-\mathbf{U}^{\mathrm{IV}}$ and $\mathbf{M}-\mathbf{U}^{\mathrm{VI}}$. 


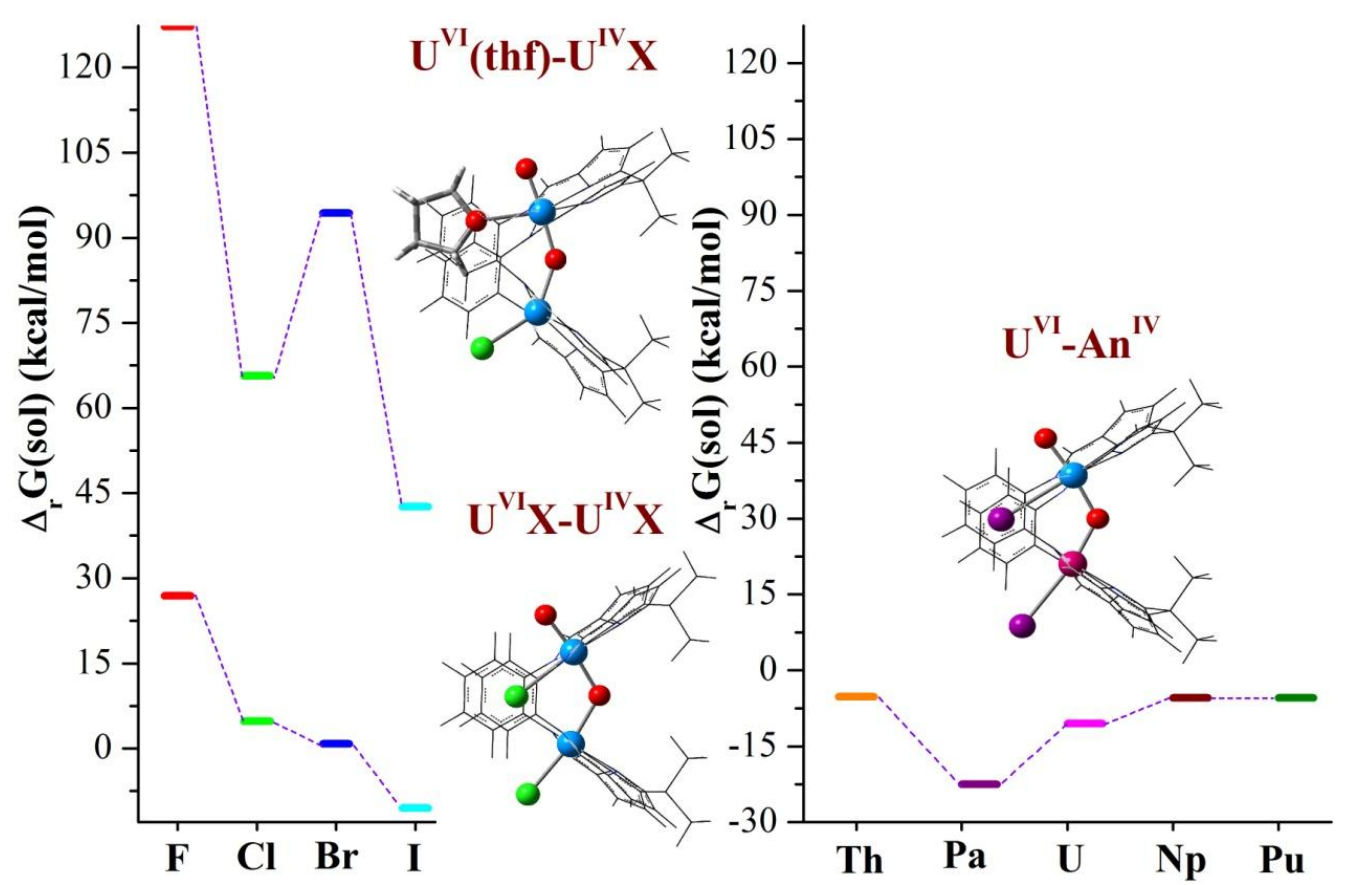

Figure S2. Free energies $\Delta_{\mathrm{r}} G(\mathrm{sol})$ of formation reactions of $\mathbf{U}^{\mathrm{VI}}(\mathbf{t h f})-\mathbf{U}^{\mathbf{I V}} \mathbf{X}, \mathbf{U}^{\mathrm{VI}} \mathbf{X}-\mathbf{U}^{\mathbf{I V}} \mathbf{X}(\mathrm{X}=\mathrm{F}, \mathrm{Cl}$, $\mathrm{Br}, \mathrm{I})$ and $\mathbf{U}^{\mathbf{V I}}-\mathbf{A n}^{\mathbf{I V}}(\mathrm{An}=\mathrm{Th}, \mathrm{Pa}, \mathrm{U}, \mathrm{Np}, \mathrm{Pu})$ 


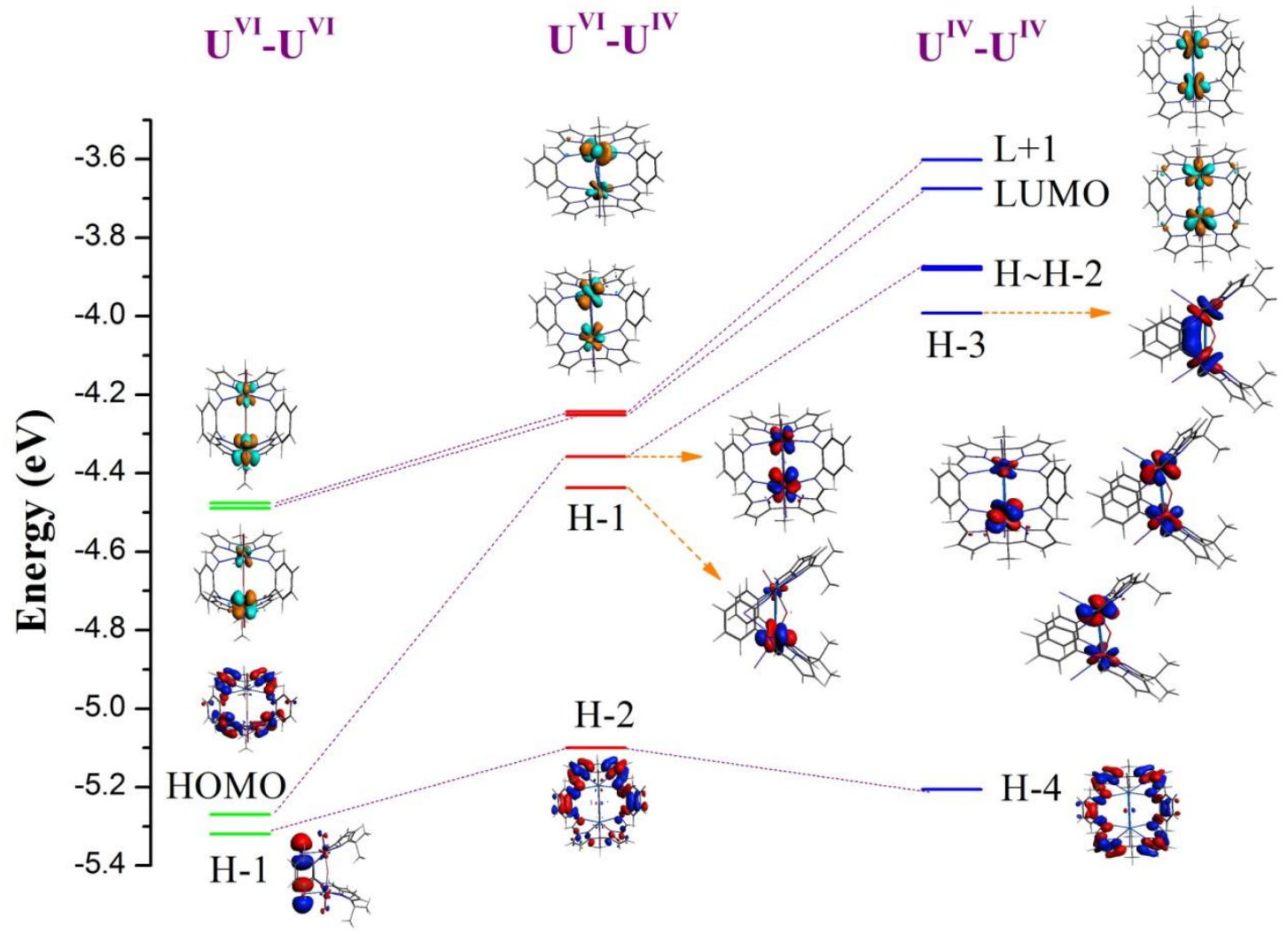

Figure S3. Energy levels and characteristic $U(f) \alpha$-spin orbitals of $\mathbf{U}^{\mathrm{VI}}-\mathbf{U}^{\mathrm{VI}}, \mathbf{U}^{\mathrm{VI}}-\mathbf{U}^{\mathrm{IV}}$ and $\mathbf{U}^{\mathrm{IV}}-\mathbf{U}^{\mathrm{IV}}$ calculated at the ADF: PBE/B-III/ZORA/COSMO level. 


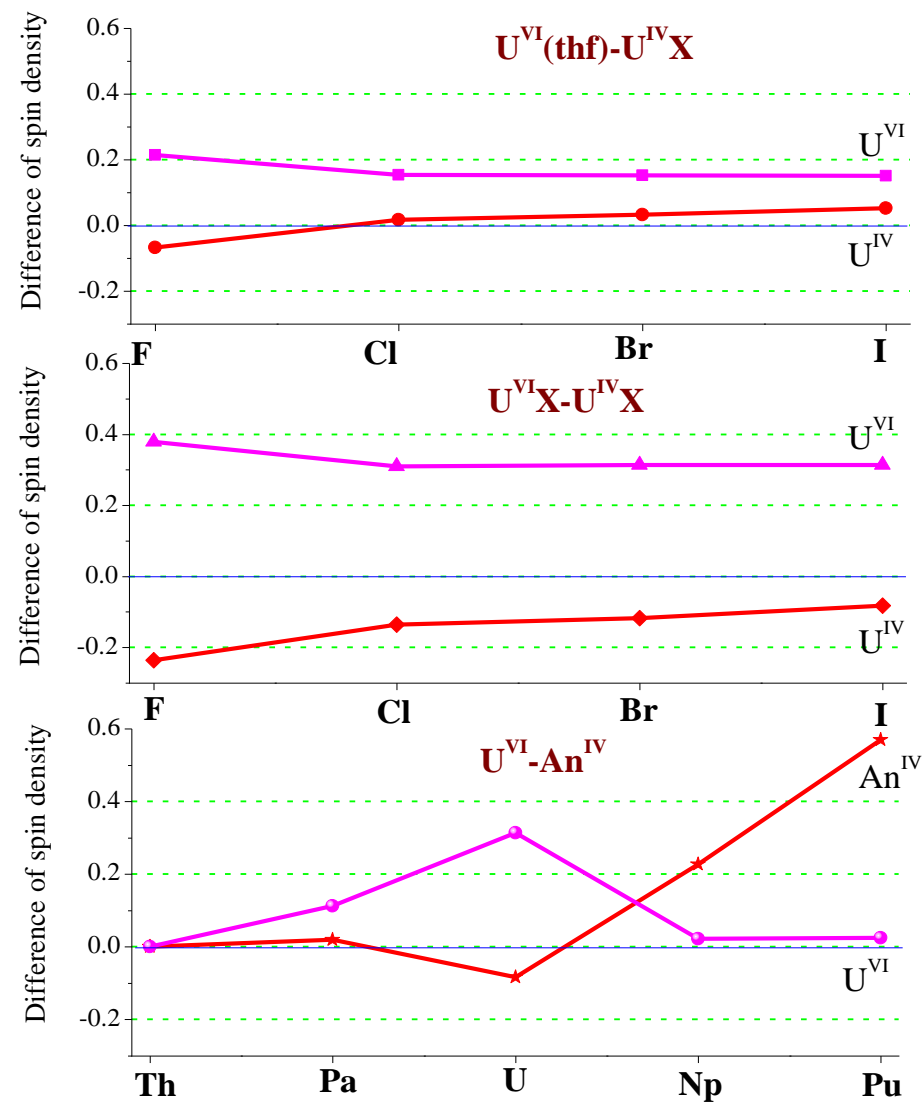

Figure S4. Difference $\left(\Delta S_{\mathrm{An}}\right)$ of spin density of actinide metal atoms for $\mathbf{U}^{\mathrm{VI}}(\mathbf{t h f})-\mathbf{U}^{\mathrm{IV}} \mathbf{X}$, $\mathbf{U}^{\mathrm{VI}} \mathbf{X}-\mathbf{U}^{\mathrm{IV}} \mathbf{X}(\mathrm{X}=\mathrm{F}, \mathrm{Cl}, \mathrm{Br}, \mathrm{I})$ and $\mathbf{U}^{\mathrm{VI}}-\mathbf{A} \mathbf{n}^{\mathbf{I V}}(\mathrm{An}=\mathrm{Th}, \mathrm{Pa}, \mathrm{U}, \mathrm{Np}, \mathrm{Pu})$ from the respective expected formal value, i.e. $0,0,1,2,3$ and 4 for $\mathrm{U}^{\mathrm{VI}}, \mathrm{Th}^{\mathrm{IV}}, \mathrm{Pa}^{\mathrm{IV}}, \mathrm{U}^{\mathrm{IV}}, \mathrm{Np}^{\mathrm{IV}}$ 和 $\mathrm{Pu}^{\mathrm{IV}}$, respectively calculated at the Priroda: PBE/B-I/AE/Gas Level. 


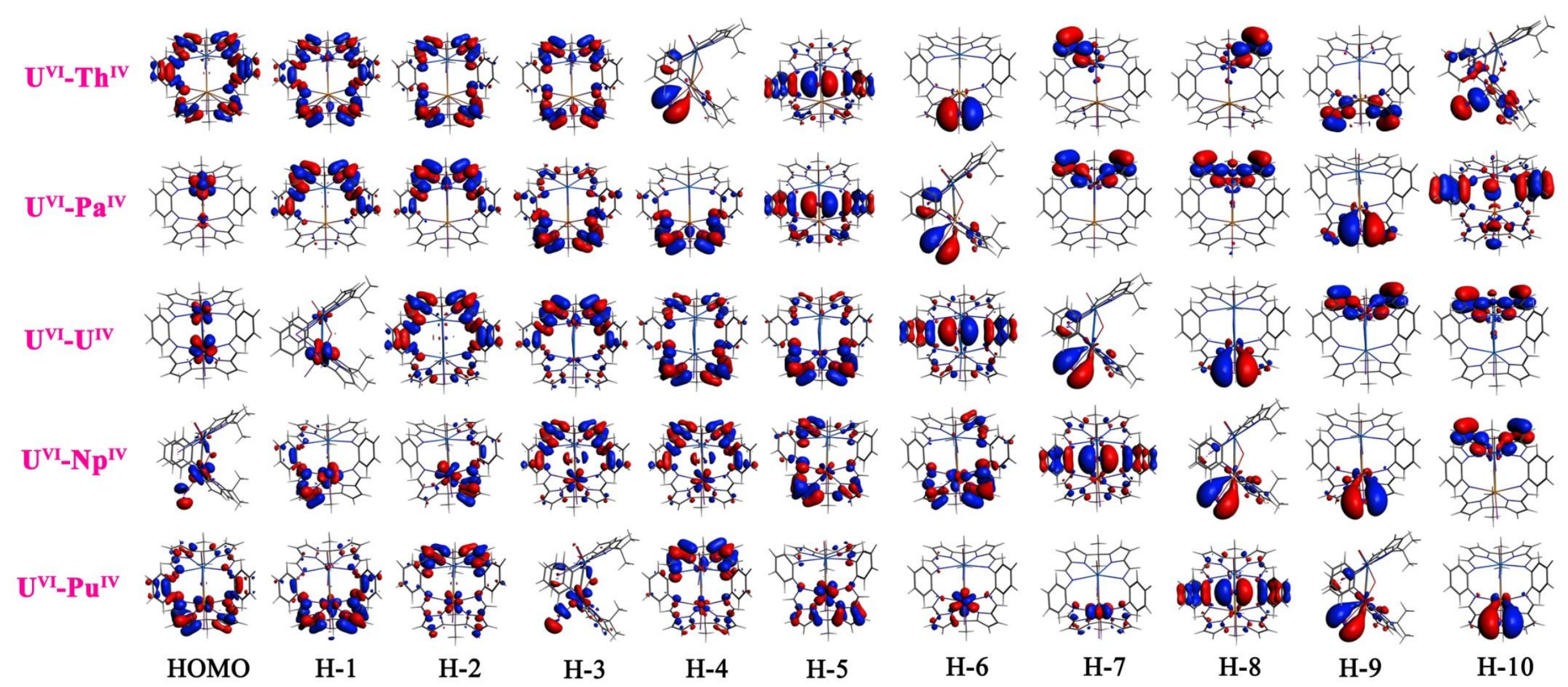

Figure S5. Diagrams of characteristic high-lying occupied $\alpha$-spin orbitals of HOMO $\sim \mathrm{H}-10$ for $\mathbf{U}^{\mathrm{VI}}-\mathbf{A n}^{\mathbf{I V}}(\mathrm{An}=\mathrm{Th}, \mathrm{Pa}, \mathrm{U}, \mathrm{Np}$ and $\mathrm{Pu})$ calculated at the ADF: PBE/B-III/ZORA/COSMO level. 


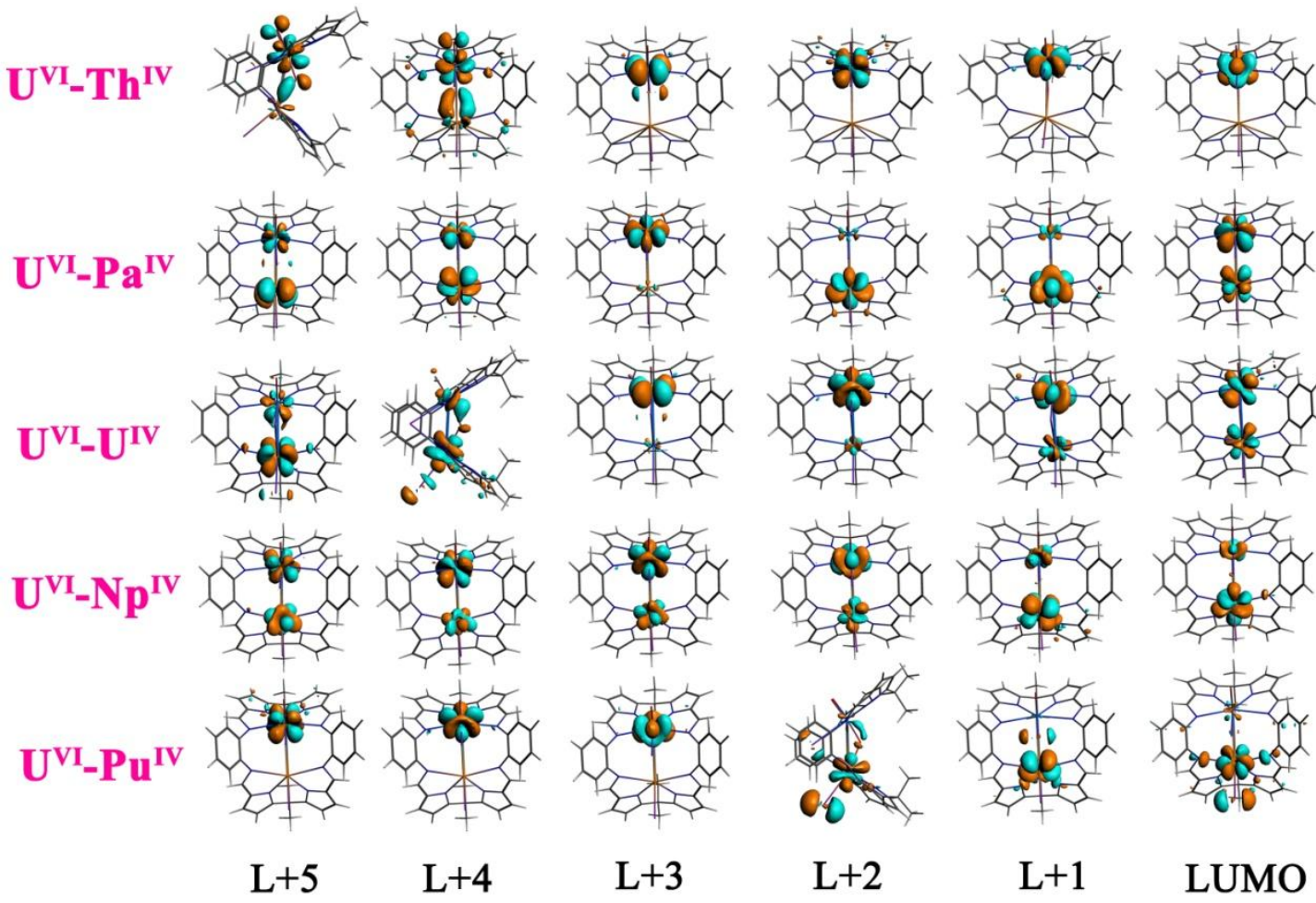

Figure S6. Diagrams of characteristic low-lying unoccupied $\alpha$-spin orbitals of L+5 LUMO for $\mathbf{U}^{\text {VI }}-\mathbf{A n}^{\text {IV }}$ (An = Th, Pa, U, Np and Pu) calculated at the ADF: PBE/B-III/ZORA/COSMO level. 


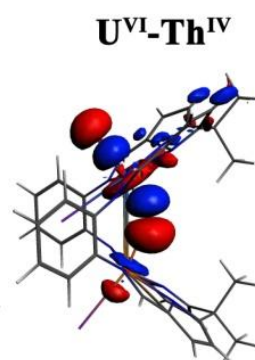

H-57

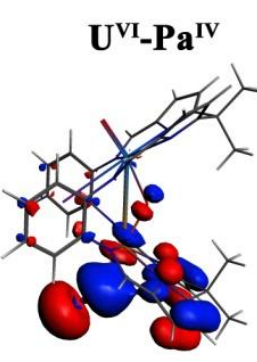

H-14

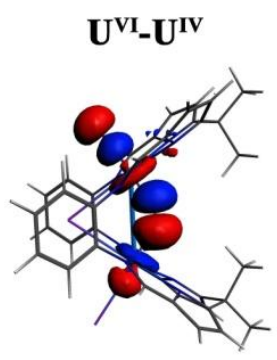

H-59

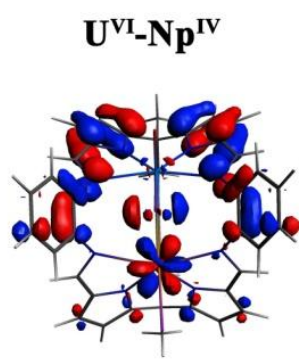

H-3

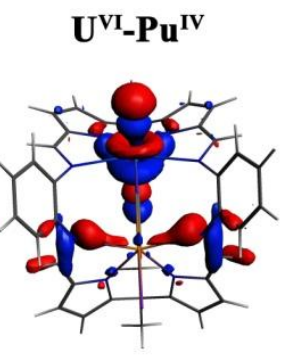

H-27

Figure S7. Diagrams of characteristic occupied $\alpha$-spin orbitals of $\mathrm{An}^{\mathrm{IV}}-\mathrm{O}_{\text {endo }}$ bond in $\mathbf{U}^{\mathrm{VI}}-\mathrm{An}^{\mathrm{IV}}$ complexes ( $\mathrm{An}=\mathrm{Th}, \mathrm{Pa}, \mathrm{U}, \mathrm{Np}$ and $\mathrm{Pu}$ ) calculated at the ADF: PBE/B-III/ZORA/COSMO level. 


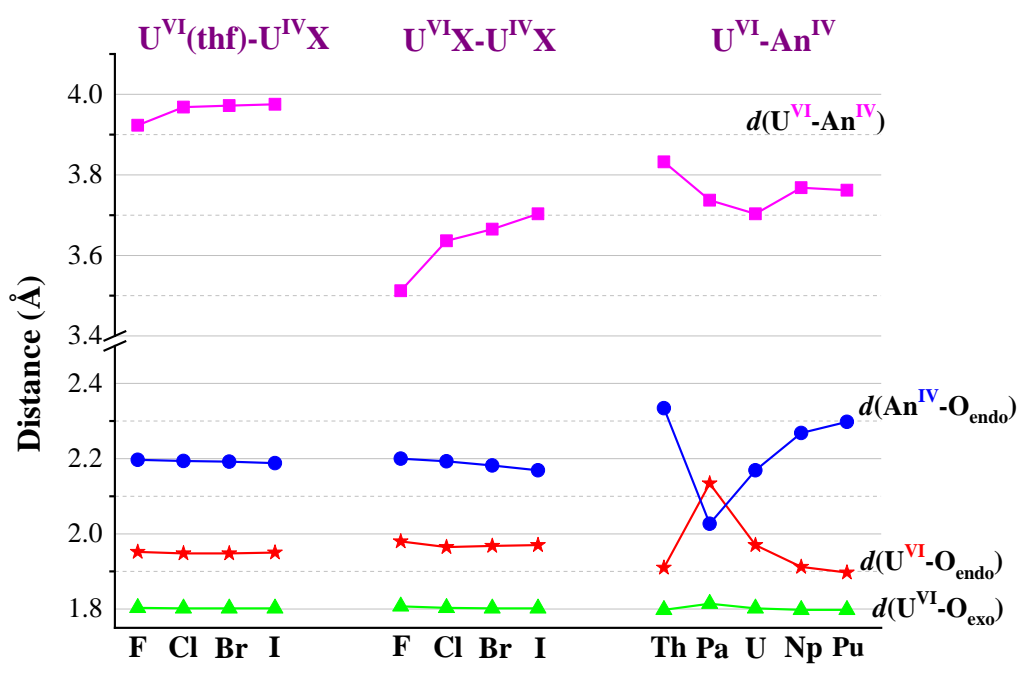

Figure S8. Optimized distances (in $\AA$ ) of $\mathbf{U}^{\mathrm{VI}}(\mathbf{t h f})-\mathbf{U}^{\mathrm{IV}} \mathbf{X}, \mathbf{U}^{\mathrm{VI}} \mathbf{X}-\mathbf{U}^{\mathbf{I V}} \mathbf{X}(\mathrm{X}=\mathrm{F}, \mathrm{Cl}, \mathrm{Br}, \mathrm{I})$ and $\mathbf{U}^{\mathrm{VI}}-\mathbf{A n}^{\mathrm{IV}}(\mathrm{An}=\mathrm{Th}, \mathrm{Pa}, \mathrm{U}, \mathrm{Np}, \mathrm{Pu})$. The pink lines represent $d\left(\mathrm{U}^{\mathrm{VI}}-\mathrm{An}^{\mathrm{IV}}\right)$, the blue lines $d\left(\mathrm{An}^{\mathrm{IV}}-\mathrm{O}_{\text {endo }}\right)$, the red lines $d\left(\mathrm{U}^{\mathrm{VI}}-\mathrm{O}_{\text {endo }}\right)$ and the green lines $d\left(\mathrm{U}^{\mathrm{VI}}-\mathrm{O}_{\text {exo }}\right)$ calculated at the Priroda: $\mathrm{PBE} / \mathrm{B}-\mathrm{I} / \mathrm{AE} / \mathrm{Gas}$ Level. 


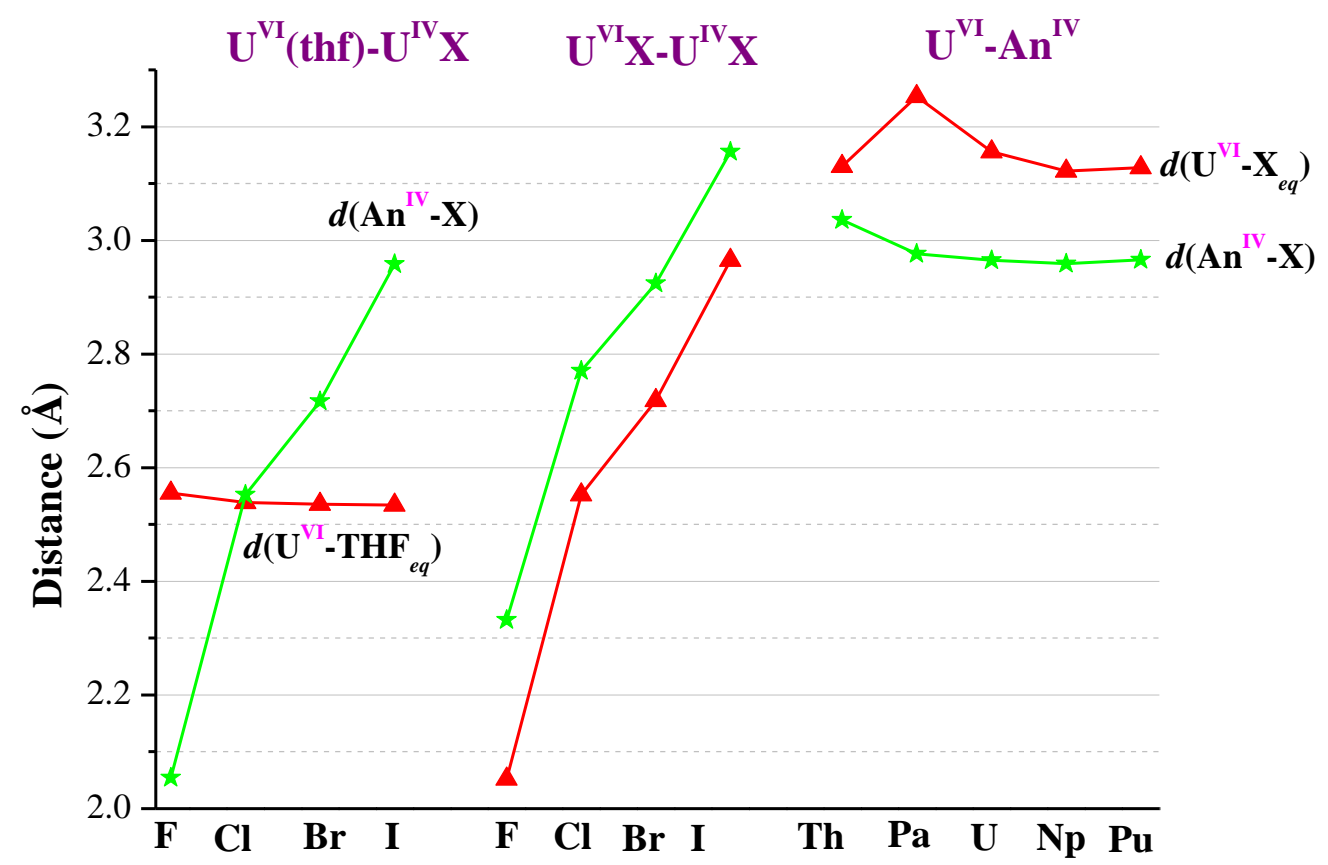

Figure S9. Optimized distances (in $\AA$ ) of $\mathbf{U}^{\mathrm{VI}}(\mathbf{t h f})-\mathbf{U}^{\mathrm{IV}} \mathbf{X}, \mathbf{U}^{\mathrm{VI}} \mathbf{X}-\mathbf{U}^{\mathrm{IV}} \mathbf{X}(\mathrm{X}=\mathrm{F}, \mathrm{Cl}, \mathrm{Br}, \mathrm{I})$ and $\mathbf{U}^{\mathrm{VI}}-\mathbf{A n}^{\mathbf{I V}}(\mathrm{An}=\mathrm{Th}, \mathrm{Pa}, \mathrm{U}, \mathrm{Np}, \mathrm{Pu})$. The red lines represent $d\left(\mathrm{U}^{\mathrm{VI}}-\mathrm{X}_{\mathrm{eq}}\right)$ and the green lines represent $d\left(\mathrm{An}^{\mathrm{IV}}-\mathrm{X}\right)$ calculated at the Priroda: $\mathrm{PBE} / \mathrm{B}-\mathrm{I} / \mathrm{AE} / \mathrm{Gas}$ Level. 


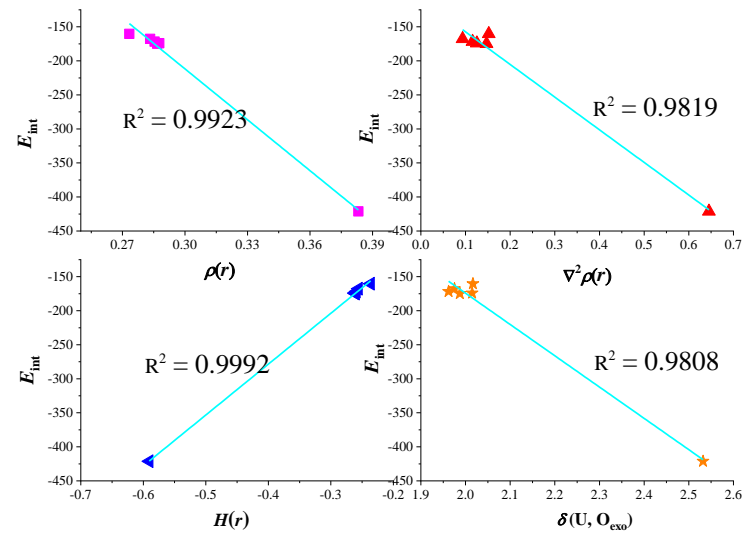

Figure S10. Plots of QTAIM parameters at the $\mathrm{U}-\mathrm{O}_{\text {exo }} \mathrm{BCP}$ and $\delta\left(\mathrm{U}, \mathrm{O}_{\text {exo }}\right)$ versus $E_{\text {int }}(\mathrm{kcal} / \mathrm{mol})$ of $\mathbf{U}^{\mathbf{V I}}-\mathbf{A n}{ }^{\mathbf{I V}}(\mathrm{An}=\mathrm{Th}, \mathrm{Pa}, \mathrm{U}, \mathrm{Np}$ and $\mathrm{Pu})$ and $\mathbf{U}^{\mathrm{VI}}(\mathbf{t h f})-\mathbf{U}^{\mathrm{IV}}(\mathbf{t h f})$. 


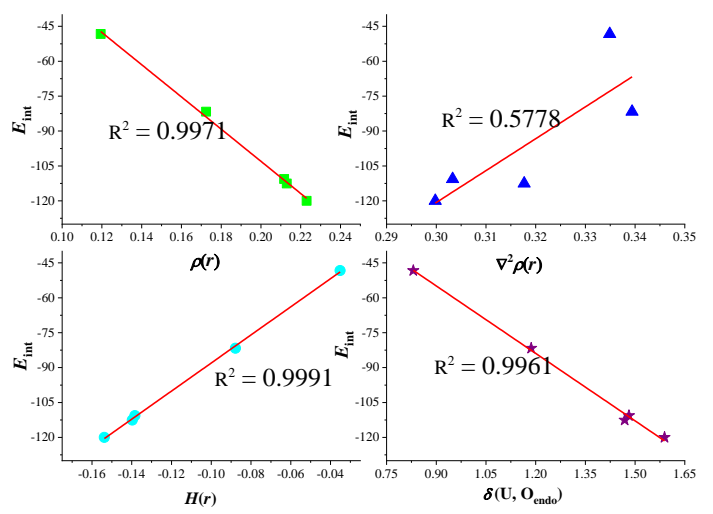

Figure S11. Plots of QTAIM parameters at the $\mathrm{U}-\mathrm{O}_{\text {endo }} \mathrm{BCP}$ and $\delta\left(\mathrm{U}, \mathrm{O}_{\text {endo }}\right)$ versus $E_{\text {int }}(\mathrm{kcal} / \mathrm{mol})$ of $\mathbf{U}^{\mathrm{VI}}-\mathbf{A n}^{\mathrm{IV}}$ (An $=\mathrm{Th}, \mathrm{Pa}, \mathrm{U}, \mathrm{Np}$ and $\left.\mathrm{Pu}\right)$ and $\mathbf{U}^{\mathrm{VI}}(\mathbf{t h f})-\mathbf{U}^{\mathrm{IV}}(\mathbf{t h f})$. 
The following reactions have been designed and calculated to form mononuclear $\left[(\mathrm{THF})\left(\mathrm{U}^{\mathrm{VI}} \mathrm{O}_{2}\right)\left(\mathrm{H}_{2} \mathrm{~L}\right)\right]\left(\mathbf{M}-\mathbf{U}^{\mathrm{VI}}\right) \cdot{ }^{1}$
$\quad \mathrm{H}_{4} \mathrm{~L}+\left[\mathrm{UO}_{2} \mathrm{I}_{2}(\mathrm{THF})_{3}\right]=\left[(\mathrm{THF})\left(\mathrm{U}^{\mathrm{VI}} \mathrm{O}_{2}\right)\left(\mathrm{H}_{2} \mathrm{~L}\right)\right]\left(\mathbf{M}-\mathbf{U}^{\mathrm{VI}}\right)+2 \mathrm{HI}+2 \mathrm{THF}$

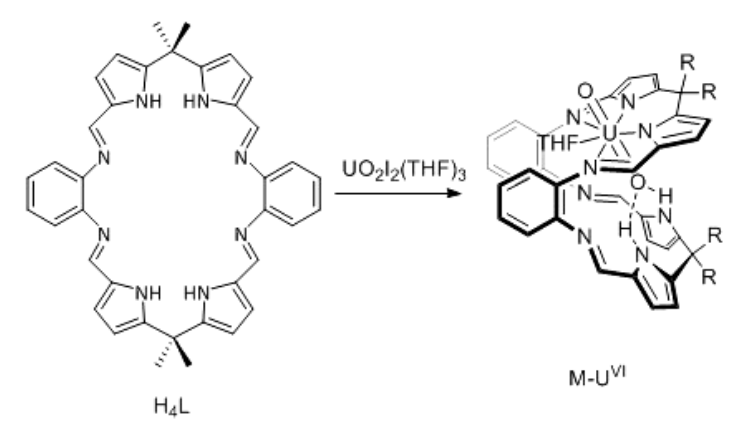

Chart S1. M-U ${ }^{\mathrm{VI}}$ complex of polypyrrolic Schiff-base macrocycles.

The calculated formation reaction energies $(\mathrm{kcal} / \mathrm{mol})$ of $\mathbf{M}-\mathbf{U}^{\mathbf{V I}}$ complex are in Table S1. It was shown that $3.2 \mathrm{kcal} / \mathrm{mol} \Delta_{\mathrm{r}} G(\mathrm{sol})$ was requried to get experimentally synthesized $\mathbf{M}-\mathbf{U}^{\mathbf{V I}}$, the spin-orbit coupling (SOC) effects were considered for all reactants and products, the $\Delta_{\mathrm{r}} G$ (sol-so) is reduced to $2.8 \mathrm{kcal} / \mathrm{mol}$ when considering spin-orbit coupling, which suggests and supports the thermodynamic feasibility for synthesizing $\mathbf{M}-\mathbf{U}^{\mathbf{V I}}$ complex.

Table S1. Calculated Formation Reaction Energies (kcal/mol) of $\left[(\mathrm{THF})\left(\mathrm{U}^{\mathrm{VI}} \mathrm{O}_{2}\right)\left(\mathrm{H}_{2} \mathrm{~L}\right)\right]\left(\mathbf{M}-\mathbf{U}^{\mathrm{VI}}\right)$ Complex.

\begin{tabular}{cccccc}
\hline$\Delta_{\mathrm{r}} E(\mathrm{gas})^{a}$ & $\Delta_{\mathrm{r}} E_{0}(\mathrm{gas})^{a}$ & $\Delta_{\mathrm{r}} G(\mathrm{gas})^{a}$ & $\Delta_{\mathrm{r}} G(\mathrm{sol}){ }^{b}$ & $\Delta_{\mathrm{r}} G_{\text {sol }}{ }^{c}$ & $\Delta_{\mathrm{r}} G_{\mathrm{so}}{ }^{d}$ \\
\hline 38.02 & 29.24 & 3.89 & 3.22 & -0.67 & -0.4 \\
\hline
\end{tabular}

${ }^{a} \Delta_{\mathrm{r}} E$ (gas), $\Delta_{\mathrm{r}} E_{0}$ (gas), $\Delta_{\mathrm{r}} H$ (gas) and $\Delta_{\mathrm{r}} G$ (gas) denote the total energy, total energy including zero-point vibration energy, enthalpy and free energy of the reaction in the gas phase, respectively.

${ }^{b} \Delta_{\mathrm{r}} G$ (sol) stands for the reaction free energy in THF solution, which includes scalar relativistic corrections and is calculated by $\Delta_{\mathrm{r}} G(\mathrm{sol})=\Delta_{\mathrm{r}} G($ gas $)+\Delta_{\mathrm{r}} G_{\text {sol }}$.

${ }^{c} \Delta_{\mathrm{r}} G_{\mathrm{sol}}=\sum v_{\mathrm{B}} G_{\mathrm{sol}}(\mathrm{B})$, where $G_{\mathrm{sol}}(\mathrm{B})$ is the calculated solvation free energies of each molecule (B) in the formation reaction, respectively.

${ }^{d} \Delta_{\mathrm{r}} G_{\mathrm{so}}=\sum v_{\mathrm{B}} G_{\mathrm{so}}(\mathrm{B})$, where $G_{\mathrm{so}}(\mathrm{B})$ is the calculated spin orbit free energies of each complex (B) in the formation reaction, respectively.

The calculated distances and angles of $\mathbf{M}-\mathbf{U}^{\mathbf{V I}}$ complex in Table S2. It was shown that the calculated geometry parameters fall well within the values of experimentally afforded. ${ }^{2}$ The optimized distances are longer than the experimental value slightly, which mainly originates from the GGA-PBE functional overestimating the bond lengths. ${ }^{3,4}$ 
Table S2. Optimized Distances (in $\AA$ ) and Angles (in degree) of $\left[(\mathrm{THF})\left(\mathrm{U}^{\mathrm{VI}} \mathrm{O}_{2}\right)\left(\mathrm{H}_{2} \mathrm{~L}\right)\right]\left(\mathbf{M}-\mathbf{U}^{\mathrm{VI}}\right)$ in the Gas Phase, Compared with Experimental Values.

\begin{tabular}{ccc}
\hline & Cal. $^{a}$ & Expt. $^{b}$ \\
\hline $\mathrm{U}^{b} \mathrm{O}_{\text {exo }}$ & 1.799 & 1.766 \\
$\mathrm{U}-\mathrm{O}_{\text {endo }}$ & 1.835 & 1.790 \\
$\mathrm{U}-\mathrm{O}_{\text {eq }}$ & 2.485 & 2.442 \\
$\mathrm{U}-\mathrm{N}$ & 2.609 & 2.593 \\
$\mathrm{U}-\mathrm{N}$ & 2.466 & 2.444 \\
$\mathrm{O}_{\text {exo }}-\mathrm{U}_{1}-\mathrm{O}_{\text {endo }}$ & 175.3 & 177.6 \\
\hline
\end{tabular}

a. Calculated values

${ }^{b .}$ Experimental values 
Catalytic one-electron reduction of uranyl(VI) to Group 1 uranyl(V) complexes via $\mathrm{Al}(\mathrm{III})$ coordination had been studied by experimental method in 2015 , the redox chemistry of $\left[\left\{(\mathrm{py})_{3} \mathrm{KOU}^{\mathrm{V}} \mathrm{O}\right\}(\mathrm{py})\left(\mathrm{H}_{2} \mathrm{~L}\right)\right]$ was studied by cyclic voltammetry in THF with $0.2 \mathrm{M}\left[\mathrm{NBu}_{4}\right]\left[\mathrm{PF}_{6}\right]$ as a supporting electrolyte at scan rates between 100 and 500 $\mathrm{mVs}^{-1}$ and reveals a quasi-reversible reduction at $\mathrm{E}_{1 / 2}=-1.31 \mathrm{~V}\left(v s . \mathrm{Fc} / \mathrm{Fc}^{+}\right)$which is ascribed to a uranyl(V)/uranyl(VI) redox couple. ${ }^{5}$

We had calculated the redox potentials of U(VI/V) pair of uranyl-exo-oxo functionalization CCI complexes, ${ }^{6}$ the results are shown in Table S1. And this time we calculated the redox potential of complex $\left[\mathrm{PaI}_{4}(\mathrm{THF})_{3}\right]$ which is ascribed to a $\mathrm{Pa}(\mathrm{V} / \mathrm{IV})$ redox couple.

The $\mathrm{U}(\mathrm{VI}) \rightarrow \mathrm{U}(\mathrm{V})$ and $\mathrm{Pa}(\mathrm{V}) \rightarrow \mathrm{Pa}(\mathrm{IV})$ single-electron reduction reaction (1) and (2) was unanimously considered to study the redox properties. Moreover, the calculated reduction potentials were modified by the spin-orbit corrections. ${ }^{7}$

$$
\begin{aligned}
& \mathbf{U}^{\mathrm{VI}} \mathbf{S o l}+e \rightarrow \mathbf{U}^{\mathrm{V}} \mathbf{S o l}(\mathbf{S o l}=\mathrm{THF}) \\
& \left(\mathbf{U}^{\mathbf{V I}} \mathbf{S o l} \mathbf{L}\right)+\mathrm{Fc} \rightarrow\left(\mathbf{U}^{\mathbf{V}} \mathbf{S o l} \mathbf{L}\right)+\mathrm{Fc}^{+} \\
& E^{0}=-\left[\Delta_{\mathrm{r}} \mathrm{G}\left(\mathrm{U}^{\mathrm{VI}} / \mathrm{U}^{\mathrm{V}}\right)-\Delta_{\mathrm{r}} \mathrm{G}\left(\mathrm{Fc}^{+} / \mathrm{Fc}\right)\right] / \mathrm{F} \\
& \mathrm{Pa}^{\mathrm{V}} \mathbf{S o l}+e \rightarrow \mathrm{Pa}^{\mathrm{IV}} \mathbf{S o l}(\mathbf{S o l}=\mathrm{THF}) \\
& \left(\mathbf{P a}^{\mathrm{V}} \text { Soll }\right)+\mathrm{Fc} \rightarrow\left(\mathbf{P a}^{\mathrm{IV}} \text { SolL }\right)+\mathrm{Fc}^{+} \\
& \mathrm{E}^{0}=-\left[\Delta_{\mathrm{r}} \mathrm{G}\left(\mathrm{Pa}^{\mathrm{V}} / \mathrm{Pa}^{\mathrm{IV}}\right)-\Delta_{\mathrm{r}} \mathrm{G}\left(\mathrm{Fc}^{+} / \mathrm{Fc}\right)\right] / \mathrm{F}
\end{aligned}
$$

Where $\mathrm{F}$ is the Faraday constant, $96485 \mathrm{C} \cdot \mathrm{mol}^{-1}$.

Table S3. Calculated Free Energies (eV) of Single-Electron Reduction Reactions ${ }^{a}$ from Uranyl(VI) to Uranyl $(\mathrm{V})$ and from $\mathrm{Pa}(\mathrm{V})$ to $\mathrm{Pa}(\mathrm{IV})$ Complexes, along with Redox Potentials $\left(\mathrm{E}^{0}\right.$ in $\mathrm{V}$ ) versus $\mathrm{Fc}^{+} / \mathrm{Fc}$ in Tetrahydrofuran (THF) Solution and Available Experimental Value.

\begin{tabular}{ccccccc}
\hline & $\mathrm{A}^{f}$ & $\mathrm{~B}^{f}$ & $\mathrm{C}^{f}$ & $\mathrm{D}^{f}$ & $\mathrm{E}^{f}$ & $\mathrm{~F}^{f}$ \\
\hline$\Delta_{\mathrm{r}} \mathrm{G}^{b}$ & -5.08 & -5.02 & -4.36 & -4.21 & -4.22 & -5.63 \\
$\Delta_{\mathrm{r}} \mathrm{G}(\mathrm{THF})^{b}$ & -4.19 & -4.12 & -3.62 & -3.46 & -3.51 & -3.94 \\
$\mathrm{E}^{0}(\mathrm{THF})^{c}$ & -1.02 & -1.09 & -1.59 & -1.75 & -1.70 & -1.27 \\
$\mathrm{E}^{0}(\mathrm{THF})_{\text {corr }}{ }^{d}$ & -0.85 & -0.91 & -1.33 & -1.50 & -1.46 & -2.98 \\
$\mathrm{E}_{1 / 2}(\mathrm{THF})^{e}$ & - & - & - & - & -1.31 & - \\
\hline
\end{tabular}

${ }^{a}$ The single-electron reduction reaction (1) and (4) as given in the text.

${ }^{b} \Delta_{\mathrm{r}} \mathrm{G}$ and $\Delta_{\mathrm{r}} \mathrm{G}$ (THF) corresponds to free energies of reaction (1) and (4) in gas phase and tetrahydrofuran (THF), respectively. $\Delta_{\mathrm{r}} \mathrm{G}(\mathrm{THF})$ of $\mathrm{Fc}^{+} / \mathrm{Fc}$ was calculated to be $-5.21 \mathrm{eV}$. 
${ }^{c} \mathrm{E}^{0}$ was calculated, according to equation (3), (6) and $\mathrm{E}^{0}$ (sol) contains solvation effects.

${ }^{d} \mathrm{E}^{0}(\mathrm{sol})_{\text {corr }}$ includes spin-orbit correction $\left(\Delta_{\mathrm{r}} \mathrm{G}_{\mathrm{so}}\right)$.

${ }^{e}$ Experimental value from the reference. ${ }^{5}$

${ }^{f}$ The A, B, C, D, E and F represent complexes and their abbreviations of $\left[\left\{\left(\mathrm{pyMe}_{2}\right) \mathrm{AlOU}^{\mathrm{V}} \mathrm{O}\right\}(\mathrm{py})\left(\mathrm{H}_{2} \mathrm{~L}\right)\right]$, $\left[\left\{\left(\right.\right.\right.$ py $\left.\left.\left.^{i} \mathrm{Bu}_{2}\right) \mathrm{AlOU}^{\mathrm{V}} \mathrm{O}\right\}(\mathrm{py})\left(\mathrm{H}_{2} \mathrm{~L}\right)\right], \quad\left[\left\{(\mathrm{py})_{3} \mathrm{LiOU}^{\mathrm{V}} \mathrm{O}\right\}(\mathrm{py})\left(\mathrm{H}_{2} \mathrm{~L}\right)\right], \quad\left[\left\{(\mathrm{py})_{3} \mathrm{NaOU}^{\mathrm{V}} \mathrm{O}\right\}(\mathrm{py})\left(\mathrm{H}_{2} \mathrm{~L}\right)\right]$, $\left[\left\{(\text { py })_{3} \mathrm{KOU}^{\mathrm{V}} \mathrm{O}\right\}(\mathrm{py})\left(\mathrm{H}_{2} \mathrm{~L}\right)\right]$ and $\left[\mathrm{Pa}^{\mathrm{IV}} \mathrm{I}_{4}(\mathrm{THF})_{3}\right]$, respectively. 
Table S4. Partial Molecular Orbital Contributions (\%) to the $\alpha$-Spin Orbitals of $\mathbf{U}^{\mathrm{VI}}-\mathbf{T h}^{\mathbf{I V}}$ Calculated at the PBE/TZP/ZORA/COSMO Level.

\begin{tabular}{|c|c|c|c|c|c|c|c|c|c|c|c|}
\hline \multirow{2}{*}{ Orbitals } & \multirow{2}{*}{$\begin{array}{c}\text { Energy } \\
(\mathrm{eV})\end{array}$} & \multicolumn{9}{|c|}{ Composition $(\%)$} & \multirow{2}{*}{ Assignment } \\
\hline & & $\mathrm{U}$ & Th & $\mathrm{O}_{e x o}$ & $\mathrm{O}_{\text {endo }}$ & $\mathrm{I}_{1}$ & $\mathrm{I}_{2}$ & $\mathrm{Ph}_{1}$ & $\mathrm{Ph}_{2}$ & $2 C^{a}$ & \\
\hline $\mathrm{L}+5$ & -3.06 & 70.89 & 3.81 & 7.78 & 5.45 & 2.09 & & & & & $\mathrm{U}(f \pi)$ \\
\hline $\mathrm{L}+4$ & -3.11 & 60.30 & 9.23 & 5.85 & 5.10 & & & & & & $\mathrm{U}(f \pi)$ \\
\hline$L+3$ & -4.24 & 89.60 & & & & 3.81 & & & & & $\mathrm{U}(f \phi)$ \\
\hline $\mathrm{L}+2$ & -4.36 & 88.98 & & & & & & & & & $\mathrm{U}(f \delta)$ \\
\hline $\mathrm{L}+1$ & -4.37 & 89.54 & & & & 1.13 & & & & & $\mathrm{U}(f \phi)$ \\
\hline LUMO & -4.39 & 92.05 & & & & & & & & & $\mathrm{U}(f \delta)$ \\
\hline HOMO & -5.15 & & & & & 2.44 & & 6.88 & 7.39 & 46.83 & \\
\hline H-1 & -5.26 & 2.19 & & & & & & 4.54 & 4.78 & 59.06 & \\
\hline $\mathrm{H}-2$ & -5.38 & & & & & & & & 1.09 & 75.25 & \\
\hline $\mathrm{H}-3$ & -5.39 & 1.19 & & & & & & & & 74.43 & \\
\hline $\mathrm{H}-4$ & -5.77 & & 4.14 & & & 5.51 & 76.92 & & & & $\mathrm{I}(p \pi)$ \\
\hline $\mathrm{H}-5$ & -5.79 & & & & & 41.13 & 1.90 & 8.16 & 8.32 & & $\mathrm{I}(p \pi)$ \\
\hline H-6 & -5.89 & & 3.29 & & & & 80.26 & & & 2.57 & $\mathrm{I}(p \pi)$ \\
\hline $\mathrm{H}-7$ & -6.17 & 1.10 & & 1.33 & & & & & & 84.19 & \\
\hline $\mathrm{H}-8$ & -6.17 & 1.05 & & 1.06 & & & & & & 84.13 & \\
\hline H-9 & -6.20 & & 1.44 & & & & 3.12 & & & 72.07 & \\
\hline $\mathrm{H}-10$ & -6.21 & & 5.07 & & & 7.48 & 41.05 & 3.07 & 3.65 & 12.41 & $\mathrm{I}(p \pi)$ \\
\hline
\end{tabular}

${ }^{a}$ The polypyrrolic ligand (L) includes two phenyl hinges ( $\left.\mathrm{Ph}\right)$ and two $\mathrm{N}_{4}$-donor compartments (2C). 
Table S5. Partial Molecular Orbital Contributions (\%) to the $\alpha$-Spin Orbitals of $\mathbf{U}^{\mathrm{VI}}-\mathbf{P a}^{\mathbf{I V}}$ Calculated at the PBE/TZP/ZORA/COSMO Level.

\begin{tabular}{|c|c|c|c|c|c|c|c|c|c|c|}
\hline \multirow{2}{*}{ Orbitals } & \multirow{2}{*}{$\begin{array}{c}\text { Energy } \\
(\mathrm{eV})\end{array}$} & \multicolumn{8}{|c|}{ Composition (\%) } & \multirow{2}{*}{ Assignment } \\
\hline & & $\mathrm{U}$ & $\mathrm{Pa}$ & $\mathrm{O}_{e x o}$ & $\mathrm{I}_{1}$ & $\mathrm{I}_{2}$ & $\mathrm{Ph}_{1}$ & $\mathrm{Ph}_{2}$ & $2 C^{a}$ & \\
\hline $\mathrm{L}+5$ & -3.65 & 13.36 & 78.93 & & 2.87 & & & & & $\mathrm{~Pa}(f \phi)+\mathrm{U}(f \delta)$ \\
\hline $\mathrm{L}+4$ & -3.68 & 21.51 & 66.02 & & & & & & & $\mathrm{~Pa}(f \phi)+\mathrm{U}(f \phi)$ \\
\hline $\mathrm{L}+3$ & -3.71 & 89.52 & 1.20 & & & & & & & $\mathrm{U}(f \pi)$ \\
\hline $\mathrm{L}+2$ & -3.73 & 1.15 & 88.87 & & & & & & & $\operatorname{Pa}(f \phi)$ \\
\hline $\mathrm{L}+1$ & -3.77 & 2.44 & 90.32 & & & & & & & $\mathrm{~Pa}(f \delta)+\mathrm{U}(f \delta)$ \\
\hline LUMO & -3.79 & 58.84 & 31.97 & & 1.83 & & & & & $\mathrm{~Pa}(f \delta)+\mathrm{U}(f \delta)$ \\
\hline HOMO & -4.07 & 91.33 & 3.23 & & & & & & & $\mathrm{U}(f \delta)$ \\
\hline H-1 & -5.07 & & & & 1.85 & & 4.61 & 5.54 & 63.66 & \\
\hline $\mathrm{H}-2$ & -5.16 & 1.81 & & & & & 1.27 & 1.18 & 69.49 & \\
\hline $\mathrm{H}-3$ & -5.41 & & & & & & 2.72 & 2.62 & 61.94 & \\
\hline $\mathrm{H}-4$ & -5.46 & & 1.19 & & & 1.08 & 2.11 & 1.10 & 66.81 & \\
\hline H-5 & -5.76 & & 1.65 & & 40.46 & & 8.20 & 7.85 & 7.78 & $\mathrm{I}(p \pi)$ \\
\hline H-6 & -5.93 & & 2.74 & & 13.56 & 59.21 & & & 2.37 & $\pi\left(\mathrm{Pa}-\mathrm{I}_{2}\right)$ \\
\hline $\mathrm{H}-7$ & -6.01 & 2.68 & & & & & & & 88.29 & \\
\hline $\mathrm{H}-8$ & -6.02 & 1.68 & & 3.36 & & 2.82 & & & 80.28 & \\
\hline H-9 & -6.10 & & 7.25 & & & 69.74 & & & 13.69 & $\pi\left(\mathrm{Pa}-\mathrm{I}_{2}\right)$ \\
\hline H-10 & -6.25 & & & & 6.50 & 4.88 & 21.82 & 17.73 & 4.75 & $\mathrm{I}(p \pi)$ \\
\hline
\end{tabular}

${ }^{a}$ The polypyrrolic ligand (L) includes two phenyl hinges ( $\left.\mathrm{Ph}\right)$ and two $\mathrm{N}_{4}$-donor compartments (2C). 
Table S6. Partial Molecular Orbital Contributions (\%) to the $\alpha$-Spin Orbitals of $\mathbf{U}^{\mathrm{VI}}-\mathbf{U}^{\mathbf{I V}}$ Calculated at the PBE/TZP/ZORA/COSMO Level.

\begin{tabular}{cccccccccc}
\hline \multirow{2}{*}{ Orbitals } & Energy & \multicolumn{7}{c}{ Composition $(\%)$} & \multirow{2}{*}{ Assignment } \\
\cline { 3 - 8 } & $(\mathrm{eV})$ & $\mathrm{U}$ & $\mathrm{U}^{b}$ & $\mathrm{I}_{1}$ & $\mathrm{I}_{2}$ & $\mathrm{Ph}_{1}$ & $\mathrm{Ph}_{2}$ & $\mathbf{2 C}^{a}$ & \\
\hline $\mathrm{L}+5$ & -3.88 & 8.63 & 77.22 & & 3.49 & & & & $\mathrm{U}(f \phi)+\mathrm{U}(f \pi)$ \\
$\mathrm{L}+4$ & -3.93 & 10.43 & 66.03 & & 6.67 & & & & $\mathrm{U}(f \sigma)+\mathrm{U}(f \sigma)$ \\
$\mathrm{L}+3$ & -4.07 & 89.18 & & 2.71 & & & & & $\mathrm{U}(f \phi)$ \\
$\mathrm{L}+2$ & -4.12 & 89.24 & 1.09 & 1.07 & & & & & $\mathrm{U}(f \delta)+\mathrm{U}(f \delta)$ \\
$\mathrm{L}+1$ & -4.24 & 81.30 & 7.87 & & & & & & \\
LUMO & -4.25 & 66.81 & 22.94 & & & & & & \\
HOMO & -4.36 & 25.05 & 61.50 & 2.38 & & & & & $\mathrm{U}(f \pi)+\mathrm{U}(f \delta)$ \\
H-1 & -4.44 & 2.40 & 87.22 & & & & & & $\mathrm{U}(f \pi)$ \\
H-2 & -5.10 & & & 2.89 & & 6.59 & 6.72 & 47.29 & \\
H-3 & -5.22 & 2.68 & 1.49 & & & 3.61 & 2.40 & 53.55 & \\
H-4 & -5.35 & & & & & 1.18 & 1.07 & 71.08 & \\
H-5 & -5.37 & & 2.35 & & & & 2.52 & 63.58 & \\
H-6 & -5.73 & 1.08 & 1.60 & 41.96 & & 8.26 & 8.03 & 2.30 & $\mathrm{I}(p \pi)$ \\
H-7 & -5.86 & & 6.74 & 7.23 & 68.78 & & & 2.79 & $\pi\left(\mathrm{U}-\mathrm{I}_{2}\right)$ \\
H-8 & -5.97 & & 7.28 & & 74.96 & & & 4.11 & $\pi\left(\mathrm{U}-\mathrm{I}_{2}\right)$ \\
H-9 & -6.08 & 4.66 & & & & & & 88.73 & \\
H-10 & -6.10 & 1.55 & & & & & 83.53 & \\
\hline
\end{tabular}

${ }^{a}$ The polypyrrolic ligand (L) includes two phenyl hinges ( $\mathrm{Ph}$ ) and two $\mathrm{N}_{4}$-donor compartments (2C).

${ }^{b} \mathrm{U}$ is for ion $\mathrm{U}^{\mathrm{IV}}$.

${ }^{c}$ The composition of exo-O and endo-O are $2.52 \%$ and $1.15 \%$.respectively. 
Table S7. Partial Molecular Orbital Contributions (\%) to the $\alpha$-Spin Orbitals of $\mathbf{U}^{\mathbf{V I}}-\mathbf{N} \mathbf{p}^{\mathbf{I V}}$ Calculated at the PBE/TZP/ZORA/COSMO Level.

\begin{tabular}{|c|c|c|c|c|c|c|c|c|c|c|}
\hline \multirow{2}{*}{ Orbitals } & \multirow{2}{*}{$\begin{array}{c}\text { Energy } \\
(\mathrm{eV})\end{array}$} & \multicolumn{8}{|c|}{ Composition (\%) } & \multirow{2}{*}{ Assignment } \\
\hline & & $\mathrm{U}$ & $\mathrm{Np}$ & $\mathrm{O}_{\text {endo }}$ & $\mathrm{I}_{1}$ & $\mathrm{I}_{2}$ & $\mathrm{Ph}_{1}$ & $\mathrm{Ph}_{2}$ & $2 C^{a}$ & \\
\hline $\mathrm{L}+5$ & -4.30 & 44.98 & 43.48 & & & & & & & $\mathrm{~Np}(f \pi)+\mathrm{U}(f \delta)$ \\
\hline $\mathrm{L}+4$ & -4.30 & 71.15 & 17.52 & & & & & & & $\mathrm{~Np}(f \delta)+\mathrm{U}(f \pi)$ \\
\hline $\mathrm{L}+3$ & -4.32 & 69.11 & 23.05 & & & & & & & $\mathrm{~Np}(f \delta)+\mathrm{U}(f \delta)$ \\
\hline $\mathrm{L}+2$ & -4.33 & 69.12 & 16.52 & & 1.84 & 1.40 & & & & $\mathrm{~Np}(f \pi)+\mathrm{U}(f \pi)$ \\
\hline $\mathrm{L}+1$ & -4.36 & 7.20 & 77.02 & & & 1.71 & & & & $\mathrm{~Np}(f \delta)+\mathrm{U}(f \delta)$ \\
\hline LUMO & -4.44 & 8.20 & 81.18 & & & & & & & $\mathrm{~Np}(f \pi)+\mathrm{U}(f \delta)$ \\
\hline HOMO & -4.86 & 1.71 & 74.26 & & 2.43 & 9.44 & & & & $\mathrm{~Np}(f \pi)$ \\
\hline H-1 & -5.03 & & 59.75 & & 2.12 & & 2.22 & & 16.99 & $\operatorname{Np}(f \delta)$ \\
\hline $\mathrm{H}-2$ & -5.07 & & 59.55 & & & & & & 15.51 & $\mathrm{~Np}(f \phi)$ \\
\hline $\mathrm{H}-3$ & -5.16 & & 23.85 & 1.03 & 5.16 & & 4.30 & 4.16 & 31.00 & $\mathrm{~Np}(f \pi)$ \\
\hline $\mathrm{H}-4$ & -5.29 & 2.93 & 11.91 & & & & 2.59 & 2.38 & 49.84 & $\mathrm{~Np}(f \phi)+\mathrm{U}(f \phi)$ \\
\hline H-5 & -5.38 & & 5.36 & & & & 2.50 & & 58.33 & $\mathrm{~Np}(f \delta)$ \\
\hline H-6 & -5.41 & & 12.05 & & & 1.08 & & 1.33 & 61.90 & $\mathrm{~Np}(f \phi)$ \\
\hline $\mathrm{H}-7$ & -5.74 & 1.37 & 2.14 & & 40.59 & & 8.37 & 7.52 & 2.25 & $\mathrm{I}(p \pi)$ \\
\hline $\mathrm{H}-8$ & -5.90 & & 10.14 & & 3.67 & 66.92 & & & 3.65 & $\pi\left(\mathrm{Np}-\mathrm{I}_{2}\right)$ \\
\hline H-9 & -5.98 & & 9.79 & & & 74.78 & & & 3.11 & $\pi\left(\mathrm{Np}-\mathrm{I}_{2}\right)$ \\
\hline $\mathrm{H}-10$ & -6.14 & 4.37 & & & & & & & 88.89 & \\
\hline
\end{tabular}

${ }^{a}$ The polypyrrolic ligand (L) includes two phenyl hinges (Ph) and two $\mathrm{N}_{4}$-donor compartments (2C). 
Table S8. Partial Molecular Orbital Contributions (\%) to the $\alpha$-Spin Orbitals of $\mathbf{U}^{\text {VI }}-\mathbf{P u}^{\text {IV }}$ Calculated at the PBE/TZP/ZORA/COSMO Level.

\begin{tabular}{|c|c|c|c|c|c|c|c|c|c|}
\hline \multirow{2}{*}{ Orbitals } & \multirow{2}{*}{$\begin{array}{c}\text { Energy } \\
(\mathrm{eV})\end{array}$} & \multicolumn{7}{|c|}{ Composition (\%) } & \multirow{2}{*}{ Assignment } \\
\hline & & $\mathrm{U}$ & $\mathrm{Pu}$ & $\mathrm{I}_{1}$ & $\mathrm{I}_{2}$ & $\mathrm{Ph}_{1}$ & $\mathrm{Ph}_{2}$ & $2 \mathrm{C}^{a}$ & \\
\hline $\mathrm{L}+5$ & -4.30 & 89.39 & & & & & & & $\mathrm{U}(f \delta)$ \\
\hline $\mathrm{L}+4$ & -4.31 & 91.40 & & 1.36 & & & & & $\mathrm{U}(f \delta)$ \\
\hline $\mathrm{L}+3$ & -4.33 & 89.88 & & 1.19 & & & & & $\mathrm{U}(f \delta)$ \\
\hline $\mathrm{L}+2$ & -4.73 & 1.23 & 72.05 & 4.28 & 13.90 & & & & $\mathrm{Pu}(f \pi)$ \\
\hline $\mathrm{L}+1$ & -4.75 & & 83.77 & 4.35 & & & & & $\operatorname{Pu}(f \phi)$ \\
\hline LUMO & -4.83 & & 66.52 & & 7.88 & & & 3.32 & $\mathrm{Pu}(f \phi)$ \\
\hline HOMO & -5.07 & & 18.61 & 1.10 & 1.24 & 5.32 & & 41.25 & $\mathrm{Pu}(f \delta)$ \\
\hline $\mathrm{H}-1$ & -5.10 & & 13.60 & & 2.90 & & 2.35 & 45.10 & $\mathrm{Pu}(f \phi)$ \\
\hline $\mathrm{H}-2$ & -5.27 & & 21.51 & & & 1.03 & & 49.01 & $\mathrm{Pu}(f \delta)$ \\
\hline $\mathrm{H}-3$ & -5.29 & 1.38 & 58.78 & 4.64 & 13.91 & & & & $\mathrm{Pu}(f \pi)+\mathrm{I}(p \pi)$ \\
\hline $\mathrm{H}-4$ & -5.34 & 2.32 & 6.10 & & & & & 60.64 & $\mathrm{Pu}(f \delta)$ \\
\hline H-5 & -5.42 & & 52.75 & & & & & 19.19 & $\operatorname{Pu}(f \delta)$ \\
\hline H-6 & -5.43 & & 79.91 & 1.59 & & & & 2.09 & $\operatorname{Pu}(f \delta)$ \\
\hline $\mathrm{H}-7$ & -5.62 & & 76.84 & 2.16 & 1.88 & & & 3.27 & $\operatorname{Pu}(f \phi)$ \\
\hline H-8 & -5.76 & 1.26 & 3.75 & 40.90 & & 9.19 & 8.50 & & \\
\hline H-9 & -5.86 & & 17.30 & 5.33 & 61.12 & & & 2.89 & $\pi\left(\mathrm{Pu}-\mathrm{I}_{2}\right)$ \\
\hline $\mathrm{H}-10$ & -5.97 & & 16.39 & & 72.36 & & & 2.48 & $\pi\left(\mathrm{Pu}-\mathrm{I}_{2}\right)$ \\
\hline
\end{tabular}

${ }^{a}$ The polypyrrolic ligand (L) includes two phenyl hinges (Ph) and two $\mathrm{N}_{4}$-donor compartments (2C). 
Table S9. Partial Molecular Orbital Contributions (\%) to the $\alpha$-Spin Orbitals of $\mathrm{An}^{\mathrm{IV}}-\mathrm{O}_{\text {endo }}$ Bond in $\mathbf{U}^{\mathrm{VI}}-\mathbf{A n}^{\mathrm{IV}}$ Complexes $(\mathrm{An}=\mathrm{Th}, \mathrm{Pa}, \mathrm{U}, \mathrm{Np}$ and $\mathrm{Pu}) \mathrm{Calculated}$ at the PBE/TZP/ZORA/COSMO Level.

\begin{tabular}{|c|c|c|c|c|c|c|c|c|}
\hline \multirow{2}{*}{ Complex } & \multirow{2}{*}{ Orbitals } & \multirow{2}{*}{$\begin{array}{c}\text { Energy } \\
(\mathrm{eV})\end{array}$} & & \multicolumn{5}{|c|}{ Composition (\%) } \\
\hline & & & An & $\mathrm{U}$ & $\mathrm{O}_{\text {endo }}$ & $\mathrm{O}_{\text {exo }}$ & $\mathrm{I}_{1}$ & $\mathbf{C}^{a}$ \\
\hline $\mathbf{U}^{\mathrm{VI}}-\mathbf{T h}^{\mathrm{IV}}$ & $\mathrm{H}-57$ & -3.662 & $1.56\left(d_{\mathrm{yz}}\right), 1.03\left(p_{\mathrm{z}}\right)$ & $7.5\left(d_{y z}, d_{z}^{2}\right)$ & $45.7\left(p_{\mathrm{z}}\right)$ & $18.5\left(p_{\mathrm{z}}, p_{\mathrm{y}}\right), 1.4(\mathrm{~s})$ & & 2.64 \\
\hline $\mathbf{U}^{\mathrm{VI}}-\mathbf{P a}^{\mathrm{IV}}$ & $\mathrm{H}-14$ & -6.441 & $4.7\left(f_{\mathrm{z} y}^{2}, f_{\mathrm{z}}^{3}, f_{\mathrm{z}}\right), 1.32\left(d_{\mathrm{z}}^{2}\right), 1.53(\mathrm{~s})$ & & $2.93\left(p_{\mathrm{z}}, p_{\mathrm{y}}\right)$ & & $38.14\left(p_{\mathrm{z}}, p_{\mathrm{y}}\right)$ & 29.92 \\
\hline $\mathbf{U}^{\mathrm{VI}}-\mathbf{U}^{\mathrm{IV}}$ & H-59 & -9.930 & $1.3\left(f_{\mathrm{z}}^{2}\right), 1.97\left(d_{y \mathrm{z}}\right), 1.08\left(p_{\mathrm{z}}\right)$ & $5.51\left(d_{y z} d_{z}^{2}\right)$ & $50.17\left(p_{\mathrm{z}}\right)$ & $16.55\left(p_{\mathrm{z}}, p_{\mathrm{y}}\right)$ & & \\
\hline $\mathrm{U}^{\mathrm{VI}}-\mathrm{Np}^{\mathrm{IV}}$ & $\mathrm{H}-3$ & -5.163 & $23.85\left(f_{\mathrm{z} x}^{2}, f_{\mathrm{xyz}}\right)$ & & $1.03\left(p_{\mathrm{x}}\right)$ & & $5.16\left(p_{\mathrm{x}}\right)$ & 38.27 \\
\hline $\mathbf{U}^{\mathrm{VI}}-\mathbf{P} \mathbf{u}^{\mathrm{IV}}$ & $\mathrm{H}-27$ & -7.782 & $1.15\left(f_{\mathrm{z}}\right), 1.12\left(d_{\mathrm{x}-\mathrm{y}}^{2}\right)$ & $23\left(f_{\mathrm{z}}^{2}, f_{\mathrm{z}}^{3}, f_{\mathrm{z}}\right), 4.38\left(p_{\mathrm{z}}\right)$ & $6.84\left(p_{\mathrm{z}}, p_{\mathrm{y}}\right)$ & $20.25\left(p_{\mathrm{z}}, p_{\mathrm{y}}\right)$ & & 10.68 \\
\hline
\end{tabular}

${ }^{a}$ The polypyrrolic ligand (L) includes two phenyl hinges $(\mathrm{Ph})$ and two $\mathrm{N}_{4}$-donor compartments $(\mathbf{C})$. 
Table S10. Optimized Distances (in $\AA$ ), Angles (in degree) and Bond Orders (in parentheses) of Bimetallic Actinide Complexes in the Gas Phase, Compared with Experimental Values of the Uranyl(VI) Complex. The theoretical approach corresponds to PRIRODA: PBE/B-I/AE/gas.

\begin{tabular}{|c|c|c|c|c|c|c|c|c|c|}
\hline Complexes & $\mathrm{U}^{\mathrm{VI}}-\mathrm{O}_{\text {exo }}$ & $\mathrm{U}^{\mathrm{VI}}-\mathrm{O}_{\text {endo }}$ & $\mathrm{U}^{\mathrm{VI}}-\mathrm{O}_{\mathrm{eq}} / \mathrm{X}_{\mathrm{eq}}$ & $\mathrm{An}^{\mathrm{IV}}-\mathrm{O}_{\text {endo }}{ }^{c}$ & $\mathrm{An}^{\mathrm{IV}}-\mathrm{X}^{c}$ & $\mathrm{U}^{\mathrm{VI}} \cdots \mathrm{An}^{\mathrm{IV} c}$ & $\alpha^{a}$ & $\beta^{a}$ & $\gamma^{a}$ \\
\hline $\mathbf{U}^{\mathrm{VI}}($ thf $)-\mathbf{U}^{\mathrm{IV}} \mathbf{F}$ & $1.803(2.44)^{b}$ & $1.952(1.53)$ & $2.555(0.40)$ & $2.197(0.79)$ & $2.054(1.27)$ & $3.923(0.40)$ & 175.6 & 141.9 & 148.6 \\
\hline $\mathbf{U}^{\mathrm{VI}}(\mathbf{t h f})-\mathbf{U}^{\mathrm{IV}} \mathbf{B r}$ & $1.802(2.44)$ & $1.948(1.55)$ & $2.536(0.41)$ & $2.192(0.41)$ & $2.717(1.31)$ & $3.972(0.32)$ & 175.7 & 147.2 & 142.5 \\
\hline $\mathbf{U}^{\mathrm{VI}} \mathbf{F}-\mathbf{U}^{\mathrm{IV}} \mathbf{F}$ & $1.807(2.44)$ & $1.980(1.46)$ & $2.332(0.58)$ & $2.200(0.87)$ & $2.052(1.27)$ & $3.512(0.61)$ & 173.7 & 114.2 & 170.4 \\
\hline $\mathbf{U}^{\mathrm{VI}} \mathbf{C l}-\mathbf{U}^{\mathrm{IV}} \mathbf{C l}$ & $1.803(2.45)$ & $1.965(1.52)$ & $2.770(0.63)$ & $2.193(0.82)$ & $2.552(1.27)$ & $3.636(0.57)$ & 173.2 & 121.8 & 170.0 \\
\hline $\mathbf{U}^{\mathrm{VI}} \mathbf{B r}-\mathbf{U}^{\mathrm{IV}} \mathbf{B r}$ & $1.802(2.45)$ & $1.968(1.51)$ & $2.924(0.66)$ & $2.182(0.83)$ & $2.718(1.30)$ & $3.665(0.56)$ & 173.1 & 124.0 & 169.8 \\
\hline $\mathbf{U}^{\mathrm{VI}}-\mathbf{P a}^{\mathrm{IV}}$ & $1.814(2.47)$ & $2.134(1.02)$ & $3.253(0.51)$ & $2.027(1.34)$ & $2.976(1.33)$ & $3.737(0.34)$ & 169.6 & 127.8 & 171.9 \\
\hline $\mathbf{U}^{\mathrm{VI}}-\mathbf{U}^{\mathrm{IV}}$ & $1.802(2.45)$ & $1.970(1.50)$ & $3.156(0.70)$ & $2.169(0.84)$ & $2.965(1.30)$ & $3.703(0.55)$ & 172.9 & 126.9 & 169.7 \\
\hline $\mathrm{U}^{\mathrm{VI}}-\mathrm{Np}^{\mathrm{IV}}$ & $1.798(2.44)$ & $1.912(1.73)$ & $3.122(0.78)$ & $2.268(0.60)$ & $2.959(1.28)$ & $3.768(0.17)$ & 173.6 & 128.4 & 169.1 \\
\hline $\mathbf{U}^{\mathrm{VI}}-\mathbf{P u}{ }^{\mathrm{IV}}$ & $1.798(2.43)$ & $1.897(1.80)$ & $3.128(0.75)$ & $2.298(0.53)$ & $2.966(1.27)$ & $3.762(0.15)$ & 173.8 & 127.2 & 164.7 \\
\hline $\mathbf{U}^{\mathrm{VI}}-\mathbf{U}^{\mathrm{VI}}$ & $1.802(2.47)$ & $2.041(1.17)$ & $2.994(1.34)$ & $2.063(1.13)$ & $2.982(1.35)$ & $4.062(0.18)$ & 179.1 & 163.7 & 100.0 \\
\hline $\mathbf{U}^{\mathrm{IV}}-\mathbf{U}^{\mathrm{IV}}$ & & $2.073(1.17)$ & $3.006(1.21)$ & $2.073(1.17)$ & $3.006(1.21)$ & $3.899(0.27)$ & & 140.2 & 148.0 \\
\hline $\operatorname{Expt} .\left(\mathrm{U}^{\mathrm{VI}}-\mathrm{Mn}\right)^{d}$ & 1.768 & 1.808 & 2.458 & 2.216 & 2.163 & 3.804 & 177.4 & 146.5 & 140.7 \\
\hline Expt. $\left(\mathrm{U}^{\mathrm{VI}}-\mathrm{Co}\right)^{d}$ & 1.771 & 1.783 & 1.783 & 2.117 & 2.084 & 3.726 & 177.8 & 148.8 & 145.6 \\
\hline
\end{tabular}

${ }^{a} \alpha, \beta$ and $\gamma$ denote angles of $\mathrm{O}_{\text {exo }}-\mathrm{U}-\mathrm{O}_{\text {endo }}$, U- $\mathrm{O}_{\text {endo }}-\mathrm{An}$ and $\mathrm{O}_{\text {endo }}-\mathrm{An}-\mathrm{X}_{\mathrm{ax}}$, respectively. ${ }^{b}$ Calculated bond orders are listed in parentheses.

${ }^{c}$ The An denotes Th, Pa, Np and Pu for complexes $\mathbf{U}^{\mathrm{VI}}-\mathbf{T} \mathbf{h}^{\mathbf{I V}}, \mathbf{U}^{\mathrm{VI}}-\mathbf{P a} \mathbf{a}^{\mathbf{I V}}, \mathbf{U}^{\mathrm{VI}}-\mathbf{N p}^{\mathbf{I V}}$ and $\mathbf{U}^{\mathrm{VI}}-\mathbf{P} \mathbf{u}^{\mathbf{I V}}$, respectively.

${ }^{d}$ Experimental values of $\mathbf{M}-\mathbf{U}^{\mathrm{VI}}\left(\left[(\mathrm{THF})\left(\mathrm{UO}_{2}\right)\left(\mathrm{H}_{2} \mathrm{~L}\right)\right]\right)^{8}$ and $[(\mathrm{THF})(\mathrm{OUOM})(\mathrm{THF})(\mathrm{L})](\mathrm{M}=\mathrm{Mn} \text { and Co })^{9}$ complexes. 
Table S11. QTAIM Data ${ }^{a}$ (au) at Various BCPs of in Bimetallic Actinide Complexes, together with the Ellipticity $\varepsilon$, Delocalization Index $\delta$ and Interaction Energy $E_{\text {int }}(\mathrm{kcal} / \mathrm{mol})$.

\begin{tabular}{|c|c|c|c|c|c|c|c|c|c|}
\hline & & $\mathrm{U}^{\mathrm{VI}}-\mathrm{Th}^{\mathrm{IV}}$ & $\mathrm{U}^{\mathrm{VI}}-\mathrm{Pa}^{\mathrm{IV}}$ & $\mathrm{U}^{\mathrm{VI}}-\mathrm{U}^{\mathrm{IV}}$ & $\mathrm{U}^{\mathrm{VI}}-\mathrm{Np}^{\mathrm{IV}}$ & $\mathrm{U}^{\mathrm{VI}}-\mathrm{Pu}^{\mathrm{IV}}$ & $\mathbf{U}^{\mathrm{VI}}(\mathbf{t h f})-\mathbf{U}^{\mathrm{IV}}(\mathbf{t h f})^{f}$ & $\mathbf{U}^{\mathrm{VI}}(\mathbf{t h f})-\mathbf{U}^{\mathrm{IV}} \mathbf{I}^{g}$ & $\mathrm{U}^{\mathrm{VI}}-\mathrm{U}^{\mathrm{IV}}$ \\
\hline \multirow[t]{8}{*}{$\mathrm{U}^{\mathrm{VI}}-\mathrm{O}_{\text {exo }}$} & $\rho(r)$ & 0.2877 & 0.2733 & 0.3832 & 0.2854 & 0.2867 & 0.2833 & 0.3982 & 0.3634 \\
\hline & $\nabla^{2} \rho(r)$ & 0.1256 & 0.1521 & 0.6451 & 0.1159 & 0.1471 & 0.0935 & 0.6360 & 0.9251 \\
\hline & $V(r)$ & -0.5548 & -0.5109 & -1.3423 & -0.5472 & -0.5573 & -0.5346 & -1.2879 & -1.3139 \\
\hline & $G(r)$ & 0.2931 & 0.2745 & 0.7518 & 0.2881 & 0.2970 & 0.2790 & 0.6766 & 0.7726 \\
\hline & $H(r)^{e}$ & -0.2617 & -0.2364 & -0.5905 & -0.2591 & -0.2602 & -0.2556 & -0.6113 & -0.5413 \\
\hline & $\delta\left(\mathrm{U}, \mathrm{O}_{\mathrm{exo}}\right)$ & 2.0149 & 2.0168 & 2.5319 & 1.9621 & 1.9874 & 1.9752 & 2.5029 & 2.2748 \\
\hline & $\varepsilon$ & 0.0183 & 0.0153 & 0.3549 & 0.0098 & 0.0139 & 0.0134 & 0.2835 & 1.1271 \\
\hline & $E_{\text {int }}$ & -174.07 & -160.30 & -421.15 & -171.69 & -174.86 & -167.73 & -404.07 & -412.25 \\
\hline \multirow[t]{8}{*}{$\mathrm{U}^{\mathrm{VI}}-\mathrm{O}_{\text {endo }}$} & $\rho(r)$ & 0.2130 & 0.1194 & $-^{h}$ & 0.2117 & 0.2230 & 0.1724 & & 0.3004 \\
\hline & $\nabla^{2} \rho(r)$ & 0.3177 & 0.3349 & ${ }^{h}$ & 0.3033 & 0.2998 & 0.3394 & & -0.1204 \\
\hline & $V(r)$ & -0.3587 & -0.1540 & ${ }^{h}$ & -0.3526 & -0.3825 & -0.2604 & & -0.8350 \\
\hline & $G(r)$ & 0.2191 & 0.1189 & $-^{h}$ & 0.2142 & 0.2287 & 0.1726 & & 0.4024 \\
\hline & $H(r)$ & -0.1397 & -0.0352 & $-^{h}$ & -0.1384 & -0.1538 & -0.0878 & & -0.4325 \\
\hline & $\delta\left(\mathrm{U}, \mathrm{O}_{\text {endo }}\right)$ & 1.4687 & 0.8308 & $-^{h}$ & 1.4814 & 1.5889 & 1.1865 & & 0.0007 \\
\hline & $\varepsilon$ & 0.0008 & 0.0375 & ${ }^{h}$ & 0.0408 & 0.0243 & 0.0107 & & 0.6277 \\
\hline & $E_{\text {int }}$ & -112.54 & -48.318 & $-^{h}$ & -110.63 & -120.01 & -81.70 & & -261.98 \\
\hline \multirow[t]{7}{*}{$\mathrm{An}^{\mathrm{IV}}-\mathrm{O}_{\text {endo }}{ }^{c}$} & $\rho(r)$ & 0.0743 & 0.1577 & 0.1306 & 0.0810 & 0.0708 & 0.1137 & 0.1201 & 0.1285 \\
\hline & $\nabla^{2} \rho(r)$ & 0.2404 & 0.3432 & 0.0791 & 0.3171 & 0.2994 & 0.3729 & 0.0869 & 0.0918 \\
\hline & $V(r)$ & -0.0796 & -0.2357 & -0.2091 & -0.0994 & -0.0844 & -0.1558 & -0.1823 & -0.2065 \\
\hline & $G(r)$ & 0.0698 & 0.1608 & 0.1144 & 0.0893 & 0.0796 & 0.1245 & 0.1020 & 0.1147 \\
\hline & $H(r)$ & -0.0097 & -0.0749 & -0.0947 & -0.0100 & -0.0048 & -0.0313 & -0.0803 & -0.0918 \\
\hline & $\delta\left(\mathrm{An}, \mathrm{O}_{\text {endo }}\right)$ & 0.5036 & 1.1888 & $\underline{-}^{h}$ & 0.4985 & 0.4362 & 0.7805 & & 1.7823 \\
\hline & $\varepsilon$ & 0.0243 & 0.0005 & 0.3165 & 0.0920 & 0.0206 & 0.1368 & 0.7758 & 0.4871 \\
\hline
\end{tabular}




\begin{tabular}{|c|c|c|c|c|c|c|c|c|c|}
\hline & $E_{\text {int }}$ & -24.98 & -73.95 & -65.61 & -31.19 & -26.48 & -48.88 & -57.19 & -64.78 \\
\hline \multirow[t]{8}{*}{$A n^{\mathrm{IV}}-\mathrm{I}_{\mathrm{ax}}{ }^{c}$} & $\rho(r)$ & 0.0497 & 0.0561 & 0.0525 & 0.0504 & 0.0471 & 0.0585 & 0.0527 & 0.0534 \\
\hline & $\nabla^{2} \rho(r)$ & 0.0574 & 0.0504 & 0.0612 & 0.0786 & 0.0866 & 0.2221 & 0.0775 & 0.0608 \\
\hline & $V(r)$ & -0.0381 & -0.0415 & -0.0414 & -0.0418 & -0.0389 & -0.0608 & -0.0425 & -0.0427 \\
\hline & $G(r)$ & 0.0262 & 0.0270 & 0.0283 & 0.0307 & 0.0303 & 0.0581 & 0.0304 & 0.0290 \\
\hline & $H(r)$ & -0.0119 & -0.0144 & -0.0131 & -0.0111 & -0.0086 & -0.0026 & -0.0121 & -0.0137 \\
\hline & $\delta\left(\mathrm{An}, \mathrm{I}_{\mathrm{ax}}\right)$ & 0.4876 & 0.9721 & 0.7409 & 0.6802 & 0.7341 & 0.3835 & & 0.7942 \\
\hline & $\varepsilon$ & 0.0068 & 0.0079 & 0.0632 & 0.0753 & 0.0394 & 0.0541 & 0.0553 & 0.0417 \\
\hline & $E_{\text {int }}$ & -11.95 & -13.02 & -12.99 & -13.12 & -12.20 & -19.08 & -13.34 & -13.40 \\
\hline \multirow[t]{8}{*}{$\mathrm{U}^{\mathrm{VI}}-\mathrm{I}_{\mathrm{eq}}$} & $\rho(r)$ & 0.0392 & 0.0303 & 0.0390 & 0.0389 & 0.0396 & 0.0438 & 0.0479 & 0.2702 \\
\hline & $\nabla^{2} \rho(r)$ & 0.0588 & 0.0543 & 0.0514 & 0.0636 & 0.0592 & 0.1648 & 0.0166 & -0.9132 \\
\hline & $V(r)$ & -0.0266 & -0.0192 & -0.0299 & -0.0275 & -0.0269 & -0.0402 & -0.1306 & -0.2978 \\
\hline & $G(r)$ & 0.0206 & 0.0164 & 0.0214 & 0.0217 & 0.0209 & 0.0407 & 0.0673 & 0.0348 \\
\hline & $H(r)$ & -0.0059 & -0.0028 & -0.0085 & -0.0058 & -0.0061 & 0.0005 & -0.0632 & -0.2631 \\
\hline & $\delta\left(\mathrm{U}, \mathrm{O}_{e q}\right)$ & 0.5781 & 0.3568 & 0.7409 & 0.5160 & 0.6070 & 0.2844 & & 0.5301 \\
\hline & $\varepsilon$ & 0.0538 & 0.1783 & 0.3685 & 0.1289 & 0.0645 & 0.0624 & 0.7820 & 0.0146 \\
\hline & $E_{\text {int }}$ & -8.35 & -6.02 & -9.38 & -8.63 & -8.44 & -12.61 & -40.96 & -93.45 \\
\hline \multirow[t]{8}{*}{$\mathrm{U}^{\mathrm{VI}}-\mathrm{N}_{\mathrm{Sch}}{ }^{d}$} & $\rho(r)$ & 0.0519 & 0.0480 & 0.0730 & 0.0505 & 0.0516 & 0.0584 & 0.0602 & 0.0532 \\
\hline & $\nabla^{2} \rho(r)$ & 0.1346 & 0.1308 & 0.1665 & 0.1430 & 0.1370 & 0.1647 & 0.1569 & 0.1432 \\
\hline & $V(r)$ & -0.0420 & -0.0390 & -0.0748 & -0.0427 & -0.0422 & -0.0535 & -0.0596 & -0.0509 \\
\hline & $G(r)$ & 0.0378 & 0.0359 & 0.0582 & 0.0392 & 0.0382 & 0.0473 & 0.0490 & 0.0434 \\
\hline & $H(r)$ & -0.0042 & -0.0032 & -0.0166 & -0.0035 & -0.0382 & -0.0061 & -0.0106 & -0.0076 \\
\hline & $\delta\left(\mathrm{U}-\mathrm{N}_{\mathrm{Sch}}\right)$ & 0.3913 & 0.3310 & 0.4686 & 0.3402 & 0.3623 & 0.3752 & & 0.3651 \\
\hline & $\varepsilon$ & 0.2410 & 0.2357 & 0.3138 & 0.2693 & 0.2194 & 0.2393 & 0.5796 & 0.3337 \\
\hline & $E_{\text {int }}$ & -13.18 & -12.24 & -23.47 & -13.40 & -13.24 & -16.79 & -18.71 & -15.98 \\
\hline $\mathrm{U}^{\mathrm{VI}}-\mathrm{N}_{\mathrm{Sch}}{ }^{c}$ & $\rho(r)$ & 0.0737 & 0.0480 & 0.0535 & 0.0516 & 0.0516 & 0.0585 & 0.0760 & 0.0529 \\
\hline
\end{tabular}




\begin{tabular}{|c|c|c|c|c|c|c|c|c|c|}
\hline & $\nabla^{2} \rho(r)$ & 0.1809 & 0.1308 & 0.1389 & 0.1460 & 0.1369 & 0.1605 & 0.1728 & 0.1440 \\
\hline & $V(r)$ & -0.0707 & -0.0390 & -0.0504 & -0.0441 & -0.0422 & -0.0525 & -0.0774 & -0.0511 \\
\hline & $G(r)$ & 0.0579 & 0.0358 & 0.0426 & 0.0403 & 0.0382 & 0.0463 & 0.0595 & 0.0436 \\
\hline & $H(r)$ & -0.0127 & -0.0032 & -0.0079 & -0.0038 & -0.0040 & -0.0062 & -0.0179 & -0.0076 \\
\hline & $\delta\left(\mathrm{U}-\mathrm{N}_{\mathrm{Sch}}\right)$ & 0.3914 & 0.3311 & 0.4806 & 0.3496 & 0.3621 & 0.3731 & & 0.3641 \\
\hline & $\varepsilon$ & 0.3149 & 0.2367 & 0.4750 & 0.2709 & 0.2192 & 0.1893 & 0.3260 & 0.4581 \\
\hline & $E_{\text {int }}$ & -22.18 & -12.24 & -15.81 & -13.84 & -13.24 & -16.47 & -24.28 & -16.05 \\
\hline \multirow[t]{8}{*}{$\mathrm{U}^{\mathrm{VI}}-\mathrm{N}_{\mathrm{pl}}{ }^{d}$} & $\rho(r)$ & 0.0737 & 0.0727 & 0.0728 & 0.0714 & 0.0729 & 0.0790 & 0.0592 & 0.0717 \\
\hline & $\nabla^{2} \rho(r)$ & 0.1809 & 0.1889 & 0.1670 & 0.1834 & 0.1808 & 0.2039 & 0.1550 & 0.1685 \\
\hline & $V(r)$ & -0.0707 & -0.0725 & -0.0747 & -0.0689 & -0.0698 & -0.0820 & -0.0585 & -0.0742 \\
\hline & $G(r)$ & 0.0580 & 0.0598 & 0.0582 & 0.0574 & 0.0575 & 0.0665 & 0.0482 & 0.0582 \\
\hline & $H(r)$ & -0.0127 & -0.0126 & -0.0165 & -0.0115 & -0.0123 & -0.0155 & -0.0103 & -0.0161 \\
\hline & $\delta\left(\mathrm{U}-\mathrm{N}_{\mathrm{pl}}\right)$ & 0.5240 & 0.5022 & 0.5989 & 0.4504 & 0.4978 & 0.4712 & & 0.4892 \\
\hline & $\varepsilon$ & 0.3148 & 0.1511 & 0.2845 & 0.2673 & 0.2866 & 0.3550 & 0.5631 & 0.3799 \\
\hline & $E_{\text {int }}$ & -22.18 & -22.75 & -23.44 & -21.62 & -21.90 & -25.73 & -18.34 & -23.29 \\
\hline \multirow[t]{8}{*}{$\mathrm{U}^{\mathrm{VI}}-\mathrm{N}_{\mathrm{pl}}^{c}$} & $\rho(r)$ & 0.0519 & 0.0727 & 0.0533 & 0.0715 & 0.0729 & 0.0794 & 0.0762 & 0.0714 \\
\hline & $\nabla^{2} \rho(r)$ & 0.1347 & 0.1890 & 0.1422 & 0.1840 & 0.1808 & 0.2080 & 0.1723 & 0.1748 \\
\hline & $V(r)$ & -0.0420 & -0.0724 & -0.0510 & -0.0691 & -0.0698 & -0.0835 & -0.0774 & -0.0756 \\
\hline & $G(r)$ & 0.0379 & 0.0598 & 0.0433 & 0.0576 & 0.0575 & 0.0677 & 0.0595 & 0.0596 \\
\hline & $H(r)$ & -0.0042 & -0.0126 & -0.0077 & -0.0116 & -0.0123 & -0.0157 & -0.0180 & -0.0159 \\
\hline & $\delta\left(\mathrm{U}-\mathrm{N}_{\mathrm{pl}}\right)$ & 0.5240 & 0.5024 & 0.6020 & 0.4512 & 0.4975 & 0.4735 & & 0.4985 \\
\hline & $\varepsilon$ & 0.2409 & 0.1482 & 0.5307 & 0.2705 & 0.2867 & 0.3931 & 0.3411 & 0.4147 \\
\hline & $E_{\text {int }}$ & -13.18 & -22.72 & -16.00 & -21.68 & -21.90 & -26.20 & -24.30 & -23.71 \\
\hline \multirow[t]{3}{*}{$\mathrm{An}^{\mathrm{IV}}-\mathrm{N}_{\mathrm{Sch}}$} & $\rho(r)$ & 0.0511 & 0.0559 & 0.0530 & 0.0500 & 0.0462 & 0.0622 & 0.0602 & 0.0537 \\
\hline & $\nabla^{2} \rho(r)$ & 0.1143 & 0.1293 & 0.1450 & 0.1522 & 0.1519 & 0.1658 & 0.1726 & 0.1420 \\
\hline & $V(r)$ & -0.0391 & -0.0434 & -0.0468 & -0.0443 & -0.0406 & -0.0580 & -0.0576 & -0.0467 \\
\hline
\end{tabular}




\begin{tabular}{|c|c|c|c|c|c|c|c|c|c|}
\hline & $G(r)$ & 0.0339 & 0.0379 & 0.0415 & 0.0412 & 0.0393 & 0.0497 & 0.0499 & 0.0411 \\
\hline & $H(r)$ & -0.0053 & -0.0056 & -0.0053 & -0.0032 & -0.0013 & -0.0083 & -0.0077 & -0.0056 \\
\hline & $\delta\left(\mathrm{An}-\mathrm{N}_{\mathrm{Sch}}\right)$ & 0.3517 & 0.4168 & 0.4201 & 0.3131 & 0.2794 & 0.4232 & & 0.4340 \\
\hline & $\varepsilon$ & 0.1530 & 0.1837 & 0.1992 & 0.1327 & 0.1314 & 0.3030 & 0.3254 & 0.1773 \\
\hline & $E_{\text {int }}$ & -12.27 & -13.62 & -14.68 & -13.90 & -12.74 & -18.20 & -18.07 & -14.67 \\
\hline \multirow[t]{8}{*}{$\mathrm{An}^{\mathrm{IV}}-\mathrm{N}_{\mathrm{Sch}}$} & $\rho(r)$ & 0.0511 & 0.0559 & 0.0516 & 0.0464 & 0.0462 & 0.0601 & 0.0551 & 0.0536 \\
\hline & $\nabla^{2} \rho(r)$ & 0.1143 & 0.1293 & 0.1445 & 0.1418 & 0.1517 & 0.1532 & 0.1625 & 0.1415 \\
\hline & $V(r)$ & -0.0391 & -0.0435 & -0.0458 & -0.0398 & -0.0405 & -0.0533 & -0.0517 & -0.0467 \\
\hline & $G(r)$ & 0.0339 & 0.0379 & 0.0410 & 0.0376 & 0.0392 & 0.0458 & 0.0458 & 0.0410 \\
\hline & $H(r)$ & -0.0053 & -0.0056 & -0.0049 & -0.0022 & -0.0013 & -0.0075 & -0.0059 & -0.0056 \\
\hline & $\delta\left(\mathrm{An}-\mathrm{N}_{\mathrm{Sch}}\right)$ & 0.3517 & 0.4161 & 0.4109 & 0.2768 & 0.2791 & 0.4301 & & 0.4350 \\
\hline & $\varepsilon$ & 0.1530 & 0.1836 & 0.3425 & 0.1448 & 0.1316 & 0.1341 & 0.4614 & 0.1540 \\
\hline & $E_{\mathrm{int}}$ & -12.27 & -13.65 & -14.37 & -12.49 & -12.71 & -16.72 & -16.22 & -14.64 \\
\hline \multirow[t]{8}{*}{$\mathrm{An}^{\mathrm{IV}}-\mathrm{N}_{\mathrm{pl}}$} & $\rho(r)$ & 0.0730 & 0.0795 & 0.0771 & 0.0770 & 0.0691 & 0.0932 & 0.0911 & 0.0787 \\
\hline & $\nabla^{2} \rho(r)$ & 0.1523 & 0.1733 & 0.1963 & 0.2256 & 0.2088 & 0.2129 & 0.2195 & 0.1869 \\
\hline & $V(r)$ & -0.0671 & -0.0744 & -0.0806 & -0.0852 & -0.0714 & -0.1032 & -0.1002 & -0.0801 \\
\hline & $G(r)$ & 0.0526 & 0.0588 & 0.0648 & 0.0708 & 0.0618 & 0.0782 & 0.0762 & 0.0634 \\
\hline & $H(r)$ & -0.0145 & -0.0155 & -0.0158 & -0.0144 & -0.0096 & -0.0250 & -0.0240 & -0.0167 \\
\hline & $\delta\left(\mathrm{An}-\mathrm{N}_{\mathrm{pl}}\right)$ & 0.4876 & 0.5643 & 0.5749 & 0.4691 & 0.4453 & 0.5800 & & 0.6036 \\
\hline & $\varepsilon$ & 0.2329 & 0.2795 & 0.2509 & 0.0156 & 0.0761 & 0.2687 & 0.1508 & 0.2252 \\
\hline & $E_{\text {int }}$ & -21.05 & -23.34 & -25.29 & -26.73 & -22.40 & -32.38 & -31.44 & -25.13 \\
\hline \multirow[t]{5}{*}{$A n^{I V}-N_{p l}$} & $\rho(r)$ & 0.0730 & 0.0796 & 0.0776 & 0.0760 & 0.0691 & 0.0933 & 0.0900 & 0.0786 \\
\hline & $\nabla^{2} \rho(r)$ & 0.1523 & 0.1733 & 0.1860 & 0.2227 & 0.2089 & 0.2237 & 0.2175 & 0.1864 \\
\hline & $V(r)$ & -0.0671 & -0.0744 & -0.0787 & -0.0836 & -0.0714 & -0.1059 & -0.0986 & -0.0798 \\
\hline & $G(r)$ & 0.0526 & 0.0589 & 0.0626 & 0.0697 & 0.0618 & 0.0809 & 0.0751 & 0.0632 \\
\hline & $H(r)$ & -0.0145 & -0.0155 & -0.0161 & -0.0140 & -0.0096 & -0.0250 & -0.0235 & -0.0166 \\
\hline
\end{tabular}




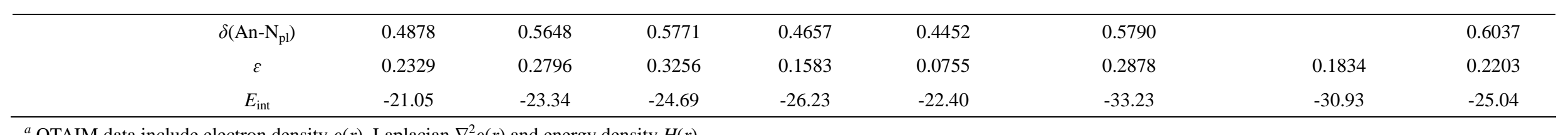

${ }^{a}$ QTAIM data include electron density $\rho(r)$, Laplacian $\nabla^{2} \rho(r)$ and energy density $H(r)$.

${ }^{b} \mathbf{T h}, \mathbf{P a}, \mathbf{U}, \mathbf{N p}, \mathbf{P u}$ and $\mathbf{U}^{\prime}$ denote complexes $\mathbf{U}^{\mathrm{VI}}-\mathbf{T h}^{\mathrm{IV}}, \mathbf{U}^{\mathrm{VI}}-\mathbf{P a}{ }^{\mathrm{IV}}, \mathbf{U}^{\mathrm{VI}}-\mathbf{U}^{\mathrm{IV}}, \mathbf{U}^{\mathrm{VI}}-\mathbf{N} \mathbf{p}^{\mathrm{IV}}, \mathbf{U}^{\mathrm{VI}}-\mathbf{P} \mathbf{u}^{\mathrm{IV}}$ and $\mathbf{U}^{\mathrm{VI}}($ thf $)-\mathbf{U}^{\mathrm{IV}}($ thf $)$, respectively.

${ }^{c}$ The An denotes Th, Pa, U, Np, Pu and U for complexes $\mathbf{U}^{\mathrm{VI}}-\mathbf{T h}^{\mathbf{I V}}, \mathbf{U}^{\mathrm{VI}}-\mathbf{P a}^{\mathbf{I V}}, \mathbf{U}^{\mathrm{VI}}-\mathbf{U}^{\mathrm{IV}}, \mathbf{U}^{\mathrm{VI}}-\mathbf{N p}^{\mathbf{I V}}, \mathbf{U}^{\mathrm{VI}}-\mathbf{P u}^{\mathbf{I V}}$ and $\mathbf{U}^{\mathrm{VI}}(\mathbf{t h f})-\mathbf{U}^{\mathrm{IV}}(\mathbf{t h f})$, respectively.

${ }^{d} \mathrm{~N}_{\mathrm{Sch}}$ and $\mathrm{N}_{\mathrm{pl}}$ denote the nitrogen atom of Schiff-base and pyrrolide, respectively.

${ }^{e} \mathrm{H}(\mathrm{r})$ is the sum of potential $V(r)$ and kinetic $G(r)$ energy density.

${ }^{f}$ The iodine atoms of complex $\mathbf{U}^{\mathrm{VI}}(\mathbf{t h f})-\mathbf{U}^{\mathrm{IV}} \mathbf{I}$ and $\mathbf{U}^{\mathrm{VI}}(\mathbf{t h f})-\mathbf{U}^{\mathbf{I V}}(\mathbf{t h f})$ in the axial and equator directions are oxygen atoms of THF, respectively.

${ }^{g}$ The base set of iodine atoms in the complex $\mathbf{U}^{\mathrm{VI}}-\mathbf{U}^{\mathbf{I V}}$ is LANL2DZ. The base sets of the iodine atoms in other complexes are LANL08D.

${ }^{h}$ There is no BCPs. 


\section{COMPUTATIONAL DETAILS}

The structures of all the complexes were optimized in the gas phase without any symmetry constraints by using the Priroda code (Version 6). ${ }^{10-13}$ A generalized gradient approximation (GGA) of perdew-Becke-ernzehoff (PBE) was applied in these calculations. An all-electron correlation-consistent Gaussian basis sets of double- $\varsigma$ polarized quality for the large component and corresponding kinetically balanced ones for the small components were utilized. ${ }^{14}$ The calculation on the $\mathbf{U}^{\mathbf{V I}}-\mathbf{U}^{\mathbf{I V}}$ for instance, includes 84 atoms and 622 electrons; the basis sets are taken as $\mathrm{U}(34 s 33 p 24 d 18 f 6 g) /(10 s 9 p 7 d 4 f 1 g), \mathrm{I}(26 s 23 p 16 d) /(6 s 5 p 3 d), \mathrm{O}(10 s 7 p 3 d) /(3 s 2 p 1 d)$, $\mathrm{N}(10 s 7 p 3 d) /(3 s 2 p 1 d), \mathrm{C}(10 s 7 p 3 d) /(3 s 2 p 1 d)$ and $\mathrm{H}(6 s 2 p) /(2 s 1 p)$, and thus, 1122 orbital basis functions with 3750 auxiliary basis functions. A scalar relativistic four-component all-electron (AE) approach was used to describe the relativistic effects. ${ }^{12,15}$ The approach arises from the full Dirac equation but with spin-orbit projected out and neglected. ${ }^{16}$ On the basis of these calculations, the optimized geometry parameters of bond distances, atomic charges (Mulliken) and bond orders (Mayer) ${ }^{17}$ were calculated.

Single point calculations have been performed by using the ADF $2014 \operatorname{code}^{18-20}$ to obtain the electronic structures and solvation energies of the complexes. A strict integration parameter of 6.0 was applied in these calculations. The solvation effects were considered by using the COSMO solvation model with THF as solvation media. $^{31,43}$ The Klamt radii were used for the main group atoms $(\mathrm{H}=1.30 \AA, \mathrm{C}=$ $2.00 \AA, \mathrm{N}=1.83 \AA, \mathrm{O}=1.72 \AA, \mathrm{F}=1.15 \AA, \mathrm{Cl}=1.40 \AA, \mathrm{Br}=1.50 \AA, \mathrm{I}=1.59 \AA$; $\mathrm{Th}$, $\mathrm{Pa}, \mathrm{U}, \mathrm{Np}$ and $\mathrm{Pu}=1.70 \AA$ ) ${ }^{1,21-25}$ The zeroth order relativistic approach $(\mathrm{ZORA})^{26-29}$ and Slater-type TZP basis sets were used in these calculations. With the small-core ZORA-TZP basis sets, the core orbitals $1 s^{-4} f$ for Th, $\mathrm{Pa}, \mathrm{U}, \mathrm{Np}$ and $\mathrm{Pu} ; 1 s^{-4 p}$ for I, $1 s^{-3 p}$ for $\mathrm{Br}, 1 s^{-}-2 p$ for $\mathrm{Cl}$ and $1 s$ for $\mathrm{F}, \mathrm{C}, \mathrm{N}$ and $\mathrm{O}$ were frozen.

To explore the bonding characteristics, we performed the quantum theory of atoms in molecule (QTAIM) at bond critical points (BCPs). ${ }^{30,31}$ This electron density-based approach has been found to be sufficiently reliable and accurate for the rationalization of actinide-ligand bonds. ${ }^{32-37}$ Firstly, single-point calculations were carried out using 
the Gaussian09 program. ${ }^{38}$ Stuttgart relativistic large-core effective core potentials (RLC-ECPs) and corresponding basis sets were applied for actinides, ${ }^{39-41}$ LANL08D for iodine atom and $6-31 \mathrm{G}^{*}$ for other atoms. Then, the electron density $\rho(r)$, laplacian electron density $\nabla^{2} \rho(r)$ and energy density $H(r)$ along with the ellipticity $(\varepsilon)$ and the delocalization index $\delta\left(\mathrm{An}, \mathrm{O}_{\text {endo }}\right)$ at $\mathrm{BCP}$ 's were computed with the Multiwfn 3.3.3 package. $^{42}$

\section{REFERENCES:}

(1) Yao, J.; Zheng, X.-J.; Pan, Q.-J.; Schreckenbach, G. Highly valence-diversified binuclear uranium complexes of a schiff-base polypyrrolic macrocycle: Prediction of unusual structures, electronic properties, and formation reactions. Inorg. Chem. 2015, 54 (11), 5438-5449.

(2) Arnold, P. L.; Blake, A. J.; Wilson, C.; Love, J. B. Uranyl complexation by a schiff-base, polypyrrolic macrocycle. Inorg. Chem. 2004, 43 (26), 8206-8.

(3) Shamov, G. A.; Schreckenbach, G.; Vo, T. N. A comparative relativistic dft and ab initio study on the structure and thermodynamics of the oxofluorides of uranium(iv), (v) and (vi). Chem. Eur. J. 2007, 13 (17), 4932-4947.

(4) Shamov, G. A.; Schreckenbach, G. Density functional studies of actinyl aquo complexes studied using small-core effective core potentials and a scalar four-component relativistic method. J. Phys. Chem. A. 2005, 109 (48), 10961-10974.

(5) Zegke, M.; Nichol, G. S.; Arnold, P. L.; Love, J. B. Catalytic one-electron reduction of uranyl(vi) to group 1 uranyl(v) complexes via al(iii) coordination. Chem. Commun. 2015, 51 (27), 5876-5879.

(6) Zheng, X.-J.; Bacha, R. U. S.; Su, D.-M.; Pan, Q.-J. Main-group metals stabilized polypyrrolic uranyl(v) complexes via cation-cation interaction with the uranyl exo-oxo atom: A relativistic density functional theory study. Inorg. Chem. 2020, 59 (24), 18018-18026.

(7) Hay, P. J.; Martin, R. L.; Schreckenbach, G. Theoretical studies of the properties and solution chemistry of ano(2)(2+) and ano(2)(+) aquo complexes for an $=u$, np, and pu. J. Phys. Chem. A. 2000, 104 (26), 6259-6270.

(8) Arnold, P. L.; Blake, A. J.; Wilson, C.; Love, J. B. Uranyl complexation by a schiff-base, polypyrrolic macrocycle. Inorg. Chem. 2004, 43 (26), 8206-8208.

(9) Arnold, P. L.; Patel, D.; Blake, A. J.; Wilson, C.; Love, J. B. Selective oxo functionalization of the uranyl ion with 3d metal cations. J. Am. Chem. Soc. 2006, 128 (30), 9610-9611.

(10) Laikov, D. N. Fast evaluation of density functional exchange-correlation terms using the expansion of the electron density in auxiliary basis sets. Chem. Phys. Lett. 1997, 281 (1-3), 151-156.

(11) Laikov, D. N. A new class of atomic basis functions for accurate electronic structure calculations of molecules. Chem. Phys. Lett. 2005, 416 (1-3), 116-120.

(12) Laikov, D. N.; Ustynyuk, Y. A. Priroda-04: A quantum-chemical program suite. New possibilities in the study of molecular systems with the application of parallel computing. Russ. Chem. Bull. 2005, 54 (3), 820-826.

(13) Laikov, D. N. Neglect of four- and approximation of one-, two-, and three-center two-electron integrals in a symmetrically orthogonalized basis. J. Comput. Chem. 2007, 28 (3), 698-702.

(14) Laikov, D. N. Ph.D. Thesis, Moscow State University, Moscow. 2000, 
(15) Laikov, D. N. An implementation of the scalar relativistic density functional theory for molecular calculatios with gaussian basis sets. DFT2000 Conference: Menton, France, 2000.

(16) Dyall, K. G. An exact separation of the spin-free and spin-dependent terms of the dirac-coulomb-breit hamiltonian. J. Chem. Phys. 1994, 100 (3), 2118-2127.

(17) Mayer, I. Simple theorems, proof and derivations in quantum chemistry. Kluwer Academic /Plenum Publishers: New York: 2003.

(18) te Velde, G.; Bickelhaupt, F. M.; Baerends, E. J.; Fonseca Guerra, C.; Van Gisbergen, S. J. A.; Snijders, J. G.; Ziegler, T. Chemistry with adf. J. Comput. Chem. 2001, 22 (9), 931-967.

(19) Fonseca Guerra, C.; Snijders, J. G.; te Velde, G.; Baerends, E. J. Towards an order-n dft method. Theor. Chem. Acc. 1998, 99 (6), 391-403.

(20) Baerends, E. J.; Ziegler, T.; Autschbach, J.; Bashford, D.; Bérces, A.; Bickelhaupt, F. M.; Bo, C.; Boerrigter, P. M.; Cavallo, L.; Chong, D. P.; Deng, L.; Dickson, R. M.; Ellis, D. E.; van Faassen, M.; Fan, L.; Fischer, T. H.; Fonseca Guerra, C.; Ghysels, A.; Giammona, A.; van Gisbergen, S. J. A.; Götz, A. W.; Groeneveld, J. A.; Gritsenko, O. V.; Grüning, M.; Gusarov, S.; Harris, F. E.; van den Hoek, P.; Jacob, C. R.; Jacobsen, H.; Jensen, L.; Kaminski, J. W.; van Kessel, G.; Kootstra, F.; Kovalenko, A.; Krykunov, M. V.; van Lenthe, E.; McCormack, D. A.; Michalak, A.; Mitoraj, M.; Neugebauer, J.; Nicu, V. P.; Noodleman, L.; Osinga, V. P.; Patchkovskii, S.; Philipsen, P. H. T.; Post, D.; Pye, C. C.; Ravenek, W.; Rodríguez, J. I.; Ros, P.; Schipper, P. R. T.; Schreckenbach, G.; Seldenthuis, J. S.; Seth, M.; Snijders, J. G.; Solà, M.; Swart, M.; Swerhone, D.; te Velde, G.; Vernooijs, P.; Versluis, L.; Visscher, L.; Visser, O.; Wang, F.; Wesolowski, T. A.; van Wezenbeek, E. M.; Wiesenekker, G.; Wolff, S. K.; Woo, T. K.; Yakovlev, A. L. Adf, ADF2010.02; SCM, Theoretical Chemistry, Vrije Universiteit: Amsterdam, The Netherlands, 2010.

(21) Klamt, A.; Jonas, V.; Burger, T.; Lohrenz, J. C. W. Refinement and parametrization of cosmo-rs. J. Phys. Chem. A. 1998, 102 (26), 5074-5085.

(22) Shamov, G. A.; Schreckenbach, G. Relativistic density functional theory study of dioxoactinide(vi) and -(v) complexation with alaskaphyrin and related schiff-base macrocyclic ligands. J. Phys. Chem. A. 2006, 110 (30), 9486-9499.

(23) Zhao, S.; Zhong, Y.; Guo, Y.; Zhang, H.; Pan, Q. A relativistic dft study of mixed oxo-imido uranium complexes of polypyrrolic macrocycle: Structure, vibrational spectrum and oxo/imido exchange reaction. Acta Chim. Sinica. 2016, 74 (8), 683-688.

(24) Bao, Z.; Zhao, H.-B.; Qu, N.; Schreckenbach, G.; Pan, Q.-J. Theoretical investigation of low-valent uranium and transuranium complexes of a flexible small-cavity macrocycle: Structural, formation reaction and redox properties. Dalton Trans. 2016, 45 (40), 15970-15982.

(25) Su, D.-M.; Zheng, X.-J.; Schreckenbach, G.; Pan, Q.-J. Highly diverse bonding between two u3+ ions when ligated by a flexible polypyrrolic macrocycle. Organometallics. 2015, 34 (21), 5225-5232.

(26) van Lenthe, E.; Ehlers, A.; Baerends, E. J. Geometry optimizations in the zero order regular approximation for relativistic effects. J. Chem. Phys. 1999, 110 (18), 8943-8953.

(27) van Lenthe, E.; Baerends, E. J.; Snijders, J. G. Relativistic total-energy using regular approximations. J. Chem. Phys. 1994, 101 (11), 9783-9792.

(28) van Lenthe, E.; Baerends, E. J.; Snijders, J. G. Relativistic regular 2-component hamiltonians. $J$. Chem. Phys. 1993, 99 (6), 4597-4610.

(29) van Lenthe, E.; Snijders, J.; Baerends, E. The zero - order regular approximation for relativistic effects: The effect of spin-orbit coupling in closed shell molecules. J. Chem. Phys. 1996, 105, 6505-6516. 
(30) Bader, R. F. W. Atoms in molecules: A quantum theory. OUP, Oxford,: 1990.

(31) Matta, C. F.; Boyd, R. J. In the quantum theory of atoms in molecules. Wiley-VCH Verlag GmbH \&Co. KGaA: Weinheim, Germany, 2007; p 1-34.

(32) Dutkiewicz, M. S.; Farnaby, J. H.; Apostolidis, C.; Colineau, E.; Walter, O.; Magnani, N.; Gardiner, M. G.; Love, J. B.; Kaltsoyannis, N.; Caciuffo, R.; Arnold, P. L. Organometallic neptunium(iii) complexes. Nat. Chem. 2016, 8 (8), 797-802.

(33) Kirker, I.; Kaltsoyannis, N. Does covalency really increase across the $5 f$ series? A comparison of molecular orbital, natural population, spin and electron density analyses of ancp 3 (an = th-cm; $\mathrm{cp}=$ [small eta]5-c5h5). Dalton Trans. 2011, 40 (1), 124-131.

(34) Huang, Q.-R.; Kingham, J. R.; Kaltsoyannis, N. The strength of actinide-element bonds from the quantum theory of atoms-in-molecules. Dalton Trans. 2015, 44 (6), 2554-2566.

(35) Chen, L.; Zheng, T.; Bao, S.; Zhang, L.; Liu, H.-K.; Zheng, L.; Wang, J.; Wang, Y.; Juan, D.; Chai, Z.; Albrecht-Schmitt, T. E.; Wang, S. A mixed-valent uranium phosphonate framework containing u-iv, u-v, and u-vi. Chem. Eur. J. 2016, 22 (34), 11954-11957.

(36) Tassell, M. J.; Kaltsoyannis, N. Covalency in ancp4 (an = th-cm): A comparison of molecular orbital, natural population and atoms-in-molecules analyses. Dalton Trans. 2010, 39 (29), 6719-6725.

(37) Wang, C.-Z.; Gibson, J. K.; Lan, J.-H.; Wu, Q.-Y.; Zhao, Y.-L.; Li, J.; Chai, Z.-F.; Shi, W.-Q. Actinide $(\mathrm{an}=\mathrm{th}-\mathrm{pu})$ dimetallocenes: Promising candidates for metal-metal multiple bonds. Dalton Trans. 2015, 44 (39), 17045-17053.

(38) Frisch, M. J.; Trucks, G. W.; Schlegel, H. B.; et. al. Gaussian 09, Gaussian 09, Revision D.01; Gaussian, Inc., Wallingford CT, 2009.

(39) Bergner, A.; Dolg, M.; Kuchle, W.; Stoll, H.; Preuss, H. Ab-initio energy-adjusted pseudopotentials for elements of groups 13-17. Mol. Phys. 1993, 80 (6), 1431-1441.

(40) Kaupp, M.; Schleyer, P. V.; Stoll, H.; Preuss, H. Pseudopotential approaches to ca, sr, and ba hydrides - why are some alkaline-earth mx2 compounds bent. J. Chem. Phys. 1991, 94 (2), 1360-1366.

(41) Dolg, M.; Stoll, H.; Preuss, H.; Pitzer, R. M. Relativistic and correlation-effects for element 105 (hahnium, ha) - a comparative-study of $\mathrm{m}$ and $\mathrm{mo}(\mathrm{m}=\mathrm{nb}$, ta, ha) using energy-adjusted abinitio pseudopotentials. J. Phys. Chem. 1993, 97 (22), 5852-5859.

(42) Lu, T.; Chen, F. Multiwfn: A multifunctional wavefunction analyzer. J. Comput. Chem. 2012, 33 (5), 580-592. 